MZ-TH/06-22

\title{
Scale-dependent metric and causal structures in Quantum Einstein Gravity
}

\author{
Martin Reuter ${ }^{1}$ and Jan-Markus Schwindt ${ }^{2}$ \\ ${ }^{1}$ Institute of Physics, University of Mainz, Staudingerweg 7, \\ D-55128 Mainz, Germany, E-mail: reuter@thep.physik.uni-mainz.de \\ 2 Institute of Theoretical Physics, University of Heidelberg, Philosophenweg 16, \\ D-69120 Heidelberg, Germany, E-mail: schwindt@thphys.uni-heidelberg.de
}

\begin{abstract}
Within the asymptotic safety scenario for gravity various conceptual issues related to the scale dependence of the metric are analyzed. The running effective field equations implied by the effective average action of Quantum Einstein Gravity (QEG) and the resulting families of resolution dependent metrics are discussed. The status of scale dependent vs. scale independent diffeomorphisms is clarified, and the difference between isometries implemented by scale dependent and independent Killing vectors is explained. A concept of scale dependent causality is proposed and illustrated by various simple examples. The possibility of assigning an "intrinsic length" to objects in a QEG spacetime is also discussed.
\end{abstract}




\section{Introduction}

During the past decade a lot of efforts went into the exploration of the nonperturbative renormalization behavior of Quantum Einstein Gravity [1]-[16]. In [1] a functional renormalization group ( $R G$ ) equation for gravity has been introduced; it defines a Wilsonian RG flow on the theory space consisting of all diffeomorphism invariant action functionals for the metric $g_{\mu \nu}$. In [1] it has been applied to the Einstein-Hilbert approximation which allows for an approximate calculation of the beta-functions of Newton's constant and the cosmological constant. The complete flow pattern was found in [4], and higher derivative truncations were analyzed in [3, 5, 10]. Matter fields were added in refs. [2, 9], and in [12] the beta-functions of [1] and [3] were used for finding optimized RG flows. The most remarkable result of these investigations is that the beta-functions of [1] predict a non-Gaussian RG fixed point [8]. After detailed studies of the reliability of the pertinent truncations [3, 4, 5, 12] it is now believed that it corresponds to a fixed point in the exact theory and is not an approximation artifact. It was found to possess all the necessary properties to make quantum gravity nonperturbatively renormalizable along the lines of Weinberg's "asymptotic safety" scenario [17, 18], thus overcoming the notorious problems related to its nonrenormalizability in perturbation theory. We shall refer to the quantum field theory of the metric tensor whose infinite cutoff limit is taken at the non-Gaussian fixed point as Quantum Einstein Gravity or "QEG". This theory should not be thought of as a quantization of classical general relativity. Its bare action is dictated by the fixed point condition and is therefore expected to contain more invariants than the Einstein-Hilbert term only. Independent evidence pointing towards a fixed point in the full theory came from the symmetry reduction approach of ref. [19] where the 2-Killing subsector of the gravitational path integral was quantized exactly.

Except for the latter investigations, all recent studies of the asymptotic safety scenario in gravity made use of the approach outlined in [1]. It is based upon the concept of the effective average action [20, 21, 22], a specific continuum implementa- 
tion of the Wilsonian renormalization group. In its original form for matter theories in flat spacetime it has been applied to a wide range of problems both in particle and statistical physics. As compared to alternative functional RG approaches in the continuum [23] the average action has various crucial advantages; the most important one is its similarity with the standard effective action $\Gamma$. In fact, the average action is a scale dependent functional $\Gamma_{k}$ depending on a "coarse graining" scale $k$ which approaches $\Gamma$ in the limit $k \rightarrow 0$ and the bare action $S$ in the limit $k \rightarrow \infty$. The close relationship of $\Gamma_{k}$ and the standard $\Gamma$ was often crucial for finding the right truncations of theory space encapsulating the essential physics. From conventional field theory we have a well-trained intuition about what a typical effective action $\Gamma$ should look like, and we can now use this experience in order to guess, and subsequently verify (or falsify) by explicit computations what the important terms in $\Gamma_{k}$ are. For the functionals evolved by the older exact RG equations a comparable understanding is lacking ususally.

Another advantage of the average action is that it defines a family of effective field theories $\left\{\Gamma_{k}, 0 \leq k<\infty\right\}$ labeled by the coarse graining scale $k$. If a physical situation involves only a single mass scale, then it is well described by a tree level evaluation of $\Gamma_{k}$, with $k$ chosen to equal that scale. In particular, the stationary points of $\Gamma_{k}$ have the interpretation of a $k$-dependent field average (approaching the standard 1-point function for $k \rightarrow 0$ ). The quality of the effective field theory description depends on the size of the fluctuations relative to the average field.

In gravity the effective average action of [1] is a diffeomorphism invariant functional of the metric: $\Gamma_{k}\left[g_{\mu \nu}\right]$. Here the analogous average field $\left\langle g_{\mu \nu}\right\rangle_{k}$ satisfies the "effective Einstein equations"

$$
\frac{\delta \Gamma_{k}}{\delta g_{\mu \nu}(x)}\left[\langle g\rangle_{k}\right]=0 .
$$

A given quantum state $|\Psi\rangle$ of the gravitational field implies an infinite family of average metrics: $\left\{\left\langle g_{\mu \nu}\right\rangle_{k}, 0 \leq k<\infty\right\}$. A scale dependence of the metric [42] has profound consequences since $\left\langle g_{\mu \nu}\right\rangle_{k}$ describes a geometry of spacetime which depends on the degree of "coarse graining", or the "resolving power" of the "mi- 
croscope" with which it is looked upon. In the case of QEG, it has been shown [3, 5] that this scale dependence leads to fractal properties of spacetime, and that in the scaling regime of the non-Gaussian fixed point, corresponding to sub-Planckian distances, the fractal dimension of spacetime equals 2. In particular, making essential use of (11) and the effective field theory properties of $\Gamma_{k}$, the spectral dimension 24] has been calculated; it was found to interpolate between 4 at macroscopic, and 2 at microscopic distances [25]. In [26], Connes et al. speculated about the possible relevance of this dimensional reduction for the noncommutative geometry of the standard model. Remarkably, exactly the same dimensional reduction has been found in Monte Carlo simulations within the causal dynamical triangulation approach [24, 27, 28].

The purpose of the present paper is to discuss in detail the conceptual status of the metric families $\left\{\left\langle g_{\mu \nu}\right\rangle_{k}, 0 \leq k<\infty\right\}$ and to illustrate, by means of simple examples, the novel physical effects which arise from a $k$-dependence of the spacetime geometry. In particular we argue that there is a well-defined notion of a scale dependent causality. We also analyze the question how, and to what extent, geometric structures or material objects in a QEG spacetime can be ascribed an "intrinsic" length which one would then consider "the" length of the objects.

The motivation for this work is that one would like to extract as much physical information as possible directly from the RG flow. Up to now this was mostly done by some form of "renormalization group improvement" [29]-38] whereby $k$ is identified with some scale typical for the physical situation under study. The notorious difficulty of this method consists in finding the correct "cutoff identification". Moreover, even if by some high degree of symmetry, for instance, this identification is uncontroversial, the disadvantage is that only spacetime properties on a single typical scale are described, albeit on a dynamically natural one. In the present paper, the idea is to completely abandon the "cutoff identification". Instead we try to "visualize" the stock of Riemannian structures $\left\{\left\langle g_{\mu \nu}\right\rangle_{k}\right\}$ as a whole and to deduce information about the physical properties of the QEG spacetimes from it. 
Before closing this introduction, let us be slightly more explicit about the construction of $\Gamma_{k}$ for gravity [1]. Its (formal) starting point is the path integral $\int \mathcal{D} \gamma_{\mu \nu} \exp (-S[\gamma])$ over all metrics $\gamma_{\mu \nu}$, gauge fixed by means of a background gauge fixing condition [39]. Even without an infrared cutoff, upon introducing sources and performing a Legendre transformation one is led to an effective action $\Gamma\left[g_{\mu \nu} ; \bar{g}_{\mu \nu}\right]$ which depends on two metrics, the expectation value of $\gamma_{\mu \nu}$, denoted $g_{\mu \nu}$, and the non-dynamical background field $\bar{g}_{\mu \nu}$. It is well-known [39] that the functional $\Gamma\left[g_{\mu \nu}\right] \equiv \Gamma\left[g_{\mu \nu} ; \bar{g}_{\mu \nu}=g_{\mu \nu}\right]$ obtained by equating the two metrics generates a possible set of 1PI Green's functions of the theory. The average action has a builtin, variable IR cutoff. This IR cutoff is implemented by first expanding the shifted integration variable $h_{\mu \nu} \equiv \gamma_{\mu \nu}-\bar{g}_{\mu \nu}$ in terms of eigenmodes of $\bar{D}^{2}$, the covariant Laplacian formed with the background metric $\bar{g}_{\mu \nu}$, and interpreting $\mathcal{D} h_{\mu \nu}$ as an integration over all expansion coefficients. Then a suppression term is introduced which damps the contribution of all $\bar{D}^{2}$-modes with eigenvalues smaller than $k^{2}$. Following the usual steps [22, 23] this leads to the scale dependent functional $\Gamma_{k}\left[g_{\mu \nu} ; \bar{g}_{\mu \nu}\right]$, and again the action with one argument is obtained by equating the two metrics: $\Gamma_{k}\left[g_{\mu \nu}\right] \equiv \Gamma_{k}\left[g_{\mu \nu} ; \bar{g}_{\mu \nu}=g_{\mu \nu}\right]$. It is this action which appears in (1).

At least when one applies the average action technique to Euclidean non-gauge theories on flat space, $\Gamma_{k}$ may be interpreted as arising from a continuum version of a Kadanoff-Wilson block spin procedure, i.e. it defines the dynamics of "coarse grained" dynamical variables which are averaged over a certain region of Euclidean spacetime. Denoting the typical linear extension of the averaging region by $\ell$, one has $\ell \approx \pi / k$ in flat spacetime. In this sense, $\Gamma_{k}$ can be thought of as a "microscope" with an adjustable resolving power $\ell=\ell(k)$. In quantum gravity where the metric is dynamical the relationship between the IR cutoff $k$ and the averaging scale $\ell$ is more complicated in general. We will return to this issue in section 3.

The running action $\Gamma_{k}$ satisfies an exact functional RG equation [1]. In practice it is usually solved on a truncated theory space. In the Einstein-Hilbert truncation 
of pure gravity $\Gamma_{k}$ is approximated by a functional of the form

$$
\Gamma_{k}[g]=(16 \pi G(k))^{-1} \int d^{4} x \sqrt{g}\{-R(g)+2 \Lambda(k)\}
$$

involving a running Newton constant $G(k)$ and cosmological constant $\Lambda(k)$. For each $k$, the action (2) implies an effective field equation which happens to be of the form of the classical Einstein equation:

$$
R_{\mu \nu}\left(\langle g\rangle_{k}\right)=\Lambda(k)\left\langle g_{\mu \nu}\right\rangle_{k}
$$

Note that the running Newton constant $G(k)$ does not appear in this effective Einstein equation. It enters only when matter fields are introduced. In this case it reads

$$
G_{\mu \nu}\left(\langle g\rangle_{k}\right)=-\Lambda(k)\left\langle g_{\mu \nu}\right\rangle_{k}+8 \pi G(k)\left\langle T_{\mu \nu}\right\rangle_{k}
$$

where the scale dependent energy momentum tensor is given by the functional derivative of the matter part of the average action, $\Gamma_{k}^{\mathrm{M}}\left[g_{\mu \nu}, \chi\right]$ :

$$
\left\langle T^{\mu \nu}(x)\right\rangle_{k} \equiv \frac{2}{\sqrt{-g}} \frac{\delta \Gamma_{k}^{\mathrm{M}}\left[\langle g\rangle_{k},\langle\chi\rangle_{k}\right]}{\delta g_{\mu \nu}(x)} .
$$

Eq. (4) is coupled to the equation of motion

$$
\frac{\delta \Gamma_{k}^{\mathrm{M}}}{\delta \chi(x)}\left[\langle g\rangle_{k},\langle\chi\rangle_{k}\right]=0
$$

Here $\chi$ denotes the set of matter fields, and $\left\{\langle g\rangle_{k},\langle\chi\rangle_{k}\right\}$ is a solution to the coupled gravity plus matter field equations. The effective Einstein equations (44) are consistent since, for $\chi$ "on shell", the energy momentum tensor is covariantly conserved:

$$
D_{\mu}\left(\langle g\rangle_{k}\right)\left\langle T^{\mu \nu}(x)\right\rangle_{k}=0
$$

Here the connection which defines the covariant derivative is built from $\langle g\rangle_{k}$. Eq. (77) is a consequence of the diffeomorphism invariance of $\Gamma_{k}^{\mathrm{M}}$.

As for the RG trajectories following from the Einstein-Hilbert approximation [4], there are several types of them, conveniently plotted in terms of the dimensionless 
parameters $g(k) \equiv k^{2} G(k)$ and $\lambda(k) \equiv \Lambda(k) / k^{2}$. Among them, "Type IIIa" trajectories are the type that is presumably realized in the real universe since it is the only type that has a positive Newton's constant $G(k)$ and a small positive cosmological constant $\Lambda(k)$ at macroscopic scales. The Type IIIa trajectory contains the following four parts, with increasing values of the cutoff $k$ :

i) The classical regime for small $k$ where the trajectory is identical to a canonical one, with $G=$ const, $\Lambda=$ const.

ii) The turnover regime where the trajectory, close to the Gaussian fixed point at $g=\lambda=0$, begins to depart from the canonical one and turns over to the separatrix which connects the Gaussian with the non-Gaussian fixed point $\left(g_{*}, \lambda_{*}\right)$. By definition, the coordinates of the turning point $T$ are $g_{T}$ and $\lambda_{T}$, and it is passed at the scale $k=k_{T}$.

iii) The growing $\Lambda$ regime where $G(k)$ is approximately constant but $\Lambda(k)$ runs proportional to $k^{4}$.

iv) The fixed point regime where the trajectory approaches the non-Gaussian fixed point in an oscillating manner. Directly at the fixed point one has $g(k) \equiv g_{*}$ and $\lambda(k) \equiv \lambda_{*}$, and therefore $G(k) \propto k^{-2}$ and $\Lambda(k) \propto k^{2}$ for $k \rightarrow \infty$. The non-Gaussian fixed point is responsible for the nonperturbative renormalizability of the theory. The behavior of the trajectory in the extreme IR is not yet known since the EinsteinHilbert approximation breaks down when $\lambda(k)$ approaches 1/2. A more general truncation is needed to approximate the RG trajectory in that region. For this reason the classical region i) does not necessarily extend to $k=0$, and we speak about "laboratory" scales for values of $k \equiv k_{\text {lab }}$ in the region where $G$ and $\Lambda$ are constant.

The remaining sections of this paper are organized as follows. In section 2 we discuss various conceptual issues related to the families $\left\{\left\langle g_{\mu \nu}\right\rangle_{k}\right\}$, in particular their connection with the quantum state, the status of $k$-independent vs. $k$-dependent diffeomorphisms, and symmetries of QEG spacetimes. In this section we also explain the idea of a scale dependent causality. Section 3 is devoted to " $k$-microscopes", a universal and mathematically simple model of an experimental setup for the obser- 
vation of the spacetime structure. The sections 4 and 5 contain various illustrative examples; in section 4 a family of Schwarzschild-de Sitter metrics is analyzed, and section 5 deals with Robertson-Walker families. In section 6 the concept of an "intrinsic scale" is described and its viability is tested in several examples. Section 7 contains a summary of the results.

At this point the reader should be warned already that the families of metrics considered in the examples do not yet correspond to realistic measurements or observations. In order to be able to find analytic solutions $\left\langle g_{\mu \nu}\right\rangle_{k}$ we usually require the spacetimes to be highly symmetric, even in presence of the "microscope". Sometimes this has the effect of overdrawing the novel effects due to the $k$-dependence of the metric so that they might appear somewhat "exotic". (In the black hole examples, for instance, the symmetry requirements amount to the assumption of a "microscope" which is much larger than the black hole itself.) As we are mostly interested in matters of principle here we shall not try to be very realistic in this respect.

\section{QEG spacetimes}

In the following we assume that we have solved the exact RG equation and picked a specific RG trajectory emanating from the non-Gaussian fixed point. This trajectory completely defines the quantum theory of the gravitational field then, in the sense that all free parameters characterizing the $\mathrm{RG}$ trajectories are given fixed values. In a standard field theory such as QED, say, this fixing of parameters corresponds to identifying the renormalized values of the electron's mass and charge with their measured values.

\subsection{State dependence}

Leaving technical issues aside for the moment [18] it should be possible to reformulate the theory resulting from a given trajectory in a Hilbert space language. In particular, one should be able to interpret the correlators of the path integral ap- 
proach, $\int \mathcal{D} \gamma \gamma_{\mu_{1} \nu_{1}}\left(x_{1}\right) \ldots \gamma_{\mu_{n} \nu_{n}}\left(x_{n}\right) \exp (-S[\gamma])$, as expectation values involving the metric operator $\hat{g}_{\mu \nu}(x)$ and a certain state $|\Psi\rangle$ :

$$
\left\langle\Psi\left|\hat{g}_{\mu_{1} \nu_{1}}\left(x_{1}\right) \ldots \hat{g}_{\mu_{n} \nu_{n}}\left(x_{n}\right)\right| \Psi\right\rangle
$$

Within the path integral formalism, the dependence on the state $|\Psi\rangle$ is encoded in the boundary conditions imposed on the fields integrated over. While this state dependence is of course central in the path integral approach applied to the elementary quantum mechanics of point particles, its importance is deemphasized in the standard matter field theories on a nondynamical Minkowski space. In the latter case $|\Psi\rangle$ is usually taken to be the essentially unique Poincaré invariant vacuum state. As there is no a priori distinguished (vacuum) state in quantum gravity we shall not try to fix $|\Psi\rangle$ here and to relate it to the boundary conditions for the path integral. However, from a conceptual point of view it will be important to keep in mind that in principle correlators such as (8) do depend on the state of the gravitational field, and that the corresponding path integral incapsulates this state dependence via boundary conditions and/or surface terms in the action (see [18] for a more detailed discussion.)

The effective average action is defined in terms of the path integral $\int \mathcal{D} \gamma_{\mu \nu} \exp (-S[\gamma])$ with additional mode suppression and source terms included. As a result, the functional $\Gamma_{k}$, too, has an implicit dependence on the state $|\Psi\rangle$.

This remark applies to the standard effective action $\Gamma=\lim _{k \rightarrow 0} \Gamma_{k}$ already. In fact since, by the usual arguments, the expectation value

$$
\left\langle\Psi\left|\hat{g}_{\mu \nu}(x)\right| \Psi\right\rangle \equiv g_{\mu \nu}(x)
$$

is a critical point of $\Gamma$,

$$
\frac{\delta \Gamma}{\delta g_{\mu \nu}}[g]=0,
$$

it is clear that $\Gamma$ "knows" about the choice for $|\Psi\rangle$. In general the effective field equations (10) will have many more solutions than just the expectation value (9). Uniqueness of the solution could be achieved by imposing subsidiary conditions on 
$g_{\mu \nu}(x)$. In principle it should be possible to derive those subsidiary conditions from the path integral over $\gamma_{\mu \nu}$.

For $k>0$, eq. (10) is replaced by the scale dependent effective field equation (10) and the situation is similar. A given $|\Psi\rangle$ translates to given boundary conditions for the path integral defining $\Gamma_{k}$. Then, by the very construction of the effective average action, the one-point function $\left\langle g_{\mu \nu}(x)\right\rangle_{k}$ defined by the cut-off path integral $\int \mathcal{D} \gamma \exp \left(-S[\gamma]-\Delta_{k} S+\cdots\right)$ is known to be a solution of (1). However, in general (1) will have many more solutions . To find out which one among them is $\left\langle g_{\mu \nu}(x)\right\rangle_{k}$, one should again restrict the space of allowed solutions by subsidiary conditions which are to be derived from the path integral and hence "know" about the state $|\Psi\rangle$.

The derivation of such subsidiary conditions is a formidable task, well beyond the present technical state of the art. In this paper we shall therefore not try to impose such conditions but rather analyze the space of all solutions to the effective field equations (of a given symmetry type), keeping in mind, however, that not all solutions necessarily come from a physically acceptable state $|\Psi\rangle$.

\subsection{Scale dependent metric structure}

Each state implies a family of mean field metrics $\left\{\left\langle g_{\mu \nu}\right\rangle_{k}(x) ; 0 \leq k<\infty\right\}$, solving the family of effective Einstein equations (1) along the chosen trajectory. As for their interpretation, it is important to note that the infinitely many equations in (19), one at each scale $k$, are valid simultaneously, and that all the mean fields $\left\langle g_{\mu \nu}\right\rangle_{k}$ refer to one and the same physical "system", a state $|\Psi\rangle$ of the "quantum spacetime" in the QEG sense. The mean fields $\left\langle g_{\mu \nu}\right\rangle_{k}$ describe the metric structure in dependence on the length scale on which the spacetime manifold is probed. An observer exploring the structure of spacetime using a "microscope" of resolution $\ell(k)$ will perceive the universe as a Riemannian manifold with the metric $\left\langle g_{\mu \nu}\right\rangle_{k}$. While $\left\langle g_{\mu \nu}\right\rangle_{k}$ is a smooth classical metric at every fixed $k$, the quantum spacetime can have fractal properties because on different scales different metrics apply. In 
this sense the metric structure on the quantum spacetime is given by an infinite set $\left\{\left\langle g_{\mu \nu}\right\rangle_{k} ; 0 \leq k<\infty\right\}$ of ordinary metrici1. Thus the picture of a "QEG spacetime" which arises from the effective field equations is that of a single differentiable manifold equipped with infinitely many Riemannian structures which are governed by the RG equations.

\section{3. $k$-independent vs. $k$-dependent diffeomorphisms}

Let us denote the manifold on which the $\left\langle g_{\mu \nu}\right\rangle_{k}$ 's are defined by $\mathcal{S}$. We interpret $\mathcal{S}$ as a dynamical spacetime and its elements $P, P^{\prime}, \cdots$ as "events". Let us first focus on the manifold structure of $\mathcal{S}$, leaving aside the Riemannian structure for a moment.

On the mathematical side, we introduce local coordinates on $\mathcal{S}$ which establish a one-to-one correspondence between points $P, P^{\prime}, \cdots$ and coordinate values $x^{\mu}(P)$, $x^{\mu}\left(P^{\prime}\right), \cdots$. On the physical side, we assume that there exists an operational procedure which allows us to identify points on $\mathcal{S}$ by means of a well-defined set of experiments. This procedure for identifying spacetime points is required to exist independently of the metric structure of $\mathcal{S}$ so that we can identify the points of $\mathcal{S}$ in a $k$-independent way. As a consequence, after having introduced coordinates, we can label the points by $x^{\mu}(P), x^{\mu}\left(P^{\prime}\right), \cdots$, and these labels are the same for all scales.

An example of a simple (thought) experiment for identifying points could be as follows. Let $\left\{\Phi^{a}(x), a=1, \ldots, 4\right\}$ be a configuration of 4 real scalar fields for which the map $x^{\mu} \mapsto \Phi^{a}$ is invertible. If this configuration is realized on $\mathcal{S}$, the result $\left(\Phi^{1}, \Phi^{2}, \Phi^{3}, \Phi^{4}\right)$ of a measurement of all scalars at the same point identifies this point uniquely2.

In the present setting the coordinate system (more precisely: the atlas) plays a much more important role than in classical gravity. It is precisely what can

\footnotetext{
${ }^{1}$ It has been shown [25] that in asymptotically safe theories of gravity, at sub-Planckian distances, spacetime is indeed a fractal [40, 41, 42, whose spectral dimension 41] equals 2.

${ }^{2}$ We are grateful to Max Niedermaier for a helpful discussion of this issue.
} 
be $k$-independently imposed on spacetime. One may visualize the whole quantum spacetime as a five-dimensional manifold, with $k$ labelling the fifth dimension. The $4 \mathrm{D}$ coordinates are used to relate the four-dimensional slices at different $k$-values with each other by saying that the point on the $\left(k=k_{1}\right)$-slice with coordinates $x^{\mu}$ is the "same" as the point on the $\left(k=k_{2}\right)$-slice with coordinates $x^{\mu}$. (This is reminiscent of the $3+1$ split in canonical gravity, where points on different spatial hypersurfaces are related via lapse and shift functions.)

The fact that both the association of coordinates to points and the physical identification of points themselves is done in a $k$-independent way has a consequence which is of crucial importance for the interpretation of the theory: It implies that the group of gauge transformations (under which "physics" is invariant) consists of $k$-independent diffeomorphisms $x^{\mu} \mapsto x^{\prime \mu}(x)$ only. As we agreed to use the same coordinate system on $\mathcal{S}$ for all scales $k, k$-dependent coordinate changes $x^{\mu} \mapsto x^{\prime \mu}(x ; k)$ are not permitted since they would alter the relationship between coordinates and physical points which had been fixed once and for all.

In the family (11) of effective field equations, $k$ plays a purely parametric role, they do not contain any derivatives with respect to $k$. The equations for different $k$-values are decoupled therefore and can be solved for each $k$ separately. Since $\Gamma_{k}[g]$ is a diffeomorphism invariant functional, the effective field equations can determine $\left\langle g_{\mu \nu}\right\rangle_{k}$ at most up to a $k$-dependent coordinate transformation. Thus, since the group of gauge transformations consists of $k$-independent transformations only, it follows that the effective field equations cannot determine the gauge invariant contents of $\left\langle g_{\mu \nu}\right\rangle_{k}$ uniquely.

The origin of this non-uniqueness is easy to understand: At one, and only one scale $k$ we have the freedom to fix a gauge, i.e. to pick any system of coordinates we like and to express the metric, at this scale, in terms of those coordinates. But after that the relationship between coordinates and physical points (events) is fixed. As a result, from the point of view of any other scale $k^{\prime} \neq k$, the coordinates have a physical meaning now and may not be changed at will any more. The 
family of field equations (11) cannot "know" about this physical interpretation the coordinates have acquired because it is covariant under a set of transformations which is infinitely much larger than the actual gauge group, namely under all $k$ dependent diffeomorphisms. This makes them "blind" to that part of the difference between $\langle g\rangle_{k}$ and $\langle g\rangle_{k^{\prime}}, k^{\prime} \neq k$, which is due to a change of coordinates. In the later sections of this paper we shall describe various examples which illustrate this phenomenon.

A different source of ambiguities which the effective field equations cannot resolve are $k$-dependent constants of integration. If, in classical gravity, a set of solutions is labeled by one real parameter, say, this parameter will be promoted to a real function of $k$. Below we shall discuss the example of the Schwarzschild mass $M$ which becomes a function $M(k)$ in quantum gravity.

If one wants to determine the metrics $\left\{\left\langle g_{\mu \nu}\right\rangle_{k} ; 0 \leq k<\infty\right\}$ unambiguously one must work with the state $|\Psi\rangle$ or the corresponding path integral directly. Given $|\Psi\rangle$ one can, at least in principle, derive a path integral whose boundary conditions encode this state, then add the mode suppression term to it, and follow the standard average action construction. Given this path integral, $\left\langle g_{\mu \nu}\right\rangle_{k}$ is unambiguously defined by $\int \mathcal{D} \gamma \gamma_{\mu \nu}(x) \exp (\cdots)$ where all metrics refer to the same coordinate system then.

\subsection{Symmetries and Killing vectors}

Let us assume we are given a family of metrics $\left\{\left\langle g_{\mu \nu}\right\rangle_{k}\right\}$, all expressed in terms of one and the same system of coordinates on $\mathcal{S}$. We can now analyze the symmetries of the Riemannian manifold $\left(\mathcal{S},\left\langle g_{\mu \nu}\right\rangle_{k}\right)$ for each value of $k$ separately. We start at some $k=k_{0}$ and search for solutions of the Killing equation

$$
\mathcal{L}_{K}\left\langle g_{\mu \nu}\right\rangle_{k}=0
$$

where $\mathcal{L}_{K}$ denotes the Lie derivative with respect to the vector field $K \equiv K^{\mu} \partial_{\mu}$. Let us assume we find a set of Killing vectors $K_{a}^{\mu}, a=1,2, \cdots$. They generate the isometry group of $\left(\mathcal{S},\left\langle g_{\mu \nu}\right\rangle_{k}\right)$ at $k=k_{0}$ in the usual way. If the scale $k_{0}$ is "generic" 
then, by continuity, we expect that $\left\langle g_{\mu \nu}\right\rangle_{k}$ will have the same isometry group also at other scales close to $k_{0}$. If $K_{a}^{\mu} \partial_{\mu}$, for $a$ fixed, is a Killing vector of $\left\langle g_{\mu \nu}\right\rangle_{k}$ on a certain $k$-interval we should distinguish the following situations:

(a) The Killing vector is the same for all values of $k$, i.e. $K_{a}^{\mu}(x) \partial_{\mu}$ is independent of $k$.

(b) The Killing vector does depend on the scale, i.e. $K_{a}^{\mu}(x ; k) \partial_{\mu}$ has an explicit parametric dependence on $k$.

In the first (second) case we say that the symmetry is implemented in a $k$-independent ( $k$-dependent) way. The motivation for this distinction is as follows. The vector field $K_{a}^{\mu}(x ; k) \partial_{\mu}$ generates a flow on $\mathcal{S}$ along which $\left\langle g_{\mu \nu}\right\rangle_{k}$ does not change. In the case (a) this flow is the same for all $k$. In view of the scale independent one-to-one correspondence between physical points and coordinates this implies that in case (a) the Killing vectors define a consistent map of physical points onto physical points.

To be more precise let us consider the two neighboring points on the same "flow line" of $K_{a}^{\mu}$ which have coordinates $x^{\mu}$ and $\bar{x}^{\mu}=x^{\mu}+\varepsilon K_{a}^{\mu}(x), \varepsilon \ll 1$, respectively. If $K_{a}^{\mu}$ is $k$-independent, the map of coordinates which it induces, $x^{\mu} \mapsto \bar{x}^{\mu}$, corresponds to a map $\mathcal{S} \rightarrow \mathcal{S}$ relating physical points. In case (b) instead, when $K_{a}^{\mu}$ depends on $k$, the target coordinate $\bar{x}^{\mu} \equiv \bar{x}^{\mu}(k)$ corresponds to different physical points for different values of $k$. Thus we see that if a group of spacetime symmetries is implemented in a $k$-dependent way it no longer corresponds to a transformation group acting on the manifold of physical events. Below we shall discuss concrete examples of both case (a) and (b), respectively. At certain (non-generic) critical values of $k$ the number of Killing vectors and their character, in particular the Lie algebra they span, can change discontinuously.

\subsection{Causal structures}

For any two events $P_{1}$ and $P_{2}$ on $\mathcal{S}$ we would like to know whether $P_{1}$ can 
influence $P_{2}$, or $P_{2}$ can influence $P_{1}$, or whether they cannot influence one another at all. The set of all such relationships between pairs of events constitutes a causal structure on $\mathcal{S}$. Within QEG this structure is, in principle, to be determined as follows.

Let us consider gravity coupled to some set of matter fields $\chi_{I}$ and let us fix some solution $\Gamma_{k}\left[g_{\mu \nu}, \chi_{I}\right]$ of the RG equation. Furthermore, we pick a solution $\left\{\langle g\rangle_{k},\left\langle\chi_{I}\right\rangle_{k} ; 0 \leq k<\infty\right\}$ of the resulting coupled effective field equations of the gravity plus matter system. This solution may be thought of as being implied by some (unperturbed) state $|\Psi\rangle \equiv\left|\Psi_{\text {gravity }}\right\rangle\left|\Psi_{\text {matter }}\right\rangle$. Next one studies perturbations about this "vacuum" state by analyzing the properties of the effective graviton propagator

$$
\left(\frac{\delta^{2} \Gamma_{k}}{\delta g_{\mu \nu}(x) \delta g_{\rho \sigma}(y)}\left[\langle g\rangle_{k},\langle\chi\rangle_{k}\right]\right)^{-1}
$$

and the effective matter field propagator

$$
\left(\frac{\delta^{2} \Gamma_{k}}{\delta \chi_{I}(x) \delta \chi_{J}(y)}\left[\langle g\rangle_{k},\langle\chi\rangle_{k}\right]\right)^{-1}
$$

Using these effective propagators one then determines the propagation characteristics of the graviton and matter field fluctuations in the given background. Knowing them, we can infer which events can be connected to a given event $P_{1}$ by a propagating gravity or matter perturbation. For each type of propagating modes one can determine a mode-dependent "causal future" of $P_{1}$, the set of events which can be influenced by $P_{1}$. Generically these sets will all be different; typically some $P_{2}$ can be in the "causal future" of $P_{1}$ with respect to one mode but not to some other.

An example in classical relativity is provided by two events on the light cone of Minkowski space: if the propagators are the classical ones, the two events can be connected by the propagating modes of the electromagnetic field, but not of some massive vector field. In quantum gravity, in particular when the renormalization effects are strong, the situation is much more involved since the effective propagators can differ quite substantially from their familiar second-order form because generically $\Gamma_{k}$ contains all sets of higher derivative and non-local terms. The graviton 
propagator in the fixed point regime [5] is of the $1 / p^{4}$-type, for instance. Moreover, it is well known that even on a fixed classical spacetime manifold matter quantum effects alter the propagation characteristics of the matter fluctuations, the photon in particular, and modify the light cone structure [43].

After having defined the mode-dependent "causal future" of $P_{1}$ it seems plausible to define the true causal future of this point as their union. Every $P_{2}$ in the true causal future of $P_{1}$ can be influenced by $P_{1}$ by at least one type of propagating mode, but typically not all of them. Analogous remarks apply to the causal past of $P_{1}$. In the standard situation with classical second-order propagators all massless fields are equally "efficient" in establishing causal links, they define the boundary of the causal future, while all massive fields are less efficient. But, as we emphasized above, RG effects can change this simple pattern.

The upshot of the above discussion is that in QEG the notion of causality is an a priori scale dependent concept. Its $k$-dependence stems from two different sources:

a) The unperturbed metric $\left\langle g_{\mu \nu}\right\rangle_{k}$ is $k$-dependent.

b) The propagation characteristics of the field perturbations which are used to send signals from one point to another are $k$-dependent.

For simplicity's sake, and in order to disentangle the two effects, we shall focus on the mechanism (a) in the present paper. We assume a situation in which the relevant propagators are still sufficiently close to the standard second-order ones so that the causal structure is determined by the light cones which are implied by the propagators of the massless fields (the photon propagator in particular, of course) or, in a geometric-optical approximation, by the null geodesics. Already in this situation a remarkable phenomenon arises: the causal structure is scale dependent because the metric $\left\langle g_{\mu \nu}\right\rangle_{k}$, and therefore the null geodesics it gives rise to, is $k$-dependent.

At first a scale-dependent causal structure might appear rather "exotic" and one might wonder whether it can lead to any logical paradoxes. However, its physical 
origin is easy to understand and a tentative interpretation would be as follows. If an event $P_{1}$ is the "cause" of an "effect" at $P_{2}$ it must be possible to send a signal from $P_{1}$ to $P_{2}$. Assuming that this signal is transmitted via some field quanta carrying energy and momentum, the signal itself influences the gravitational field. In general it will also be modified by the physical (i.e. gravitating) apparatuses used as a "transmitter" and "receiver". Within the effective field theory approach the dominant modifications can be taken into account by changing the scale at which $\Gamma_{k}$ is evaluated; the new $k$-value should take into account the typical scale set by the signal transmission process. Whether or not this is a quantitatively good approximation depends on how well the transmission of the signal can be modeled by a single-scale process. Nevertheless, in priciple it is conceivable that different experimental setups consisting of a transmitter, the signal, and a receiver, involve different typical scales (sizes, momenta, virtualities, etc.) and "see" different average metrics $\left\langle g_{\mu \nu}\right\rangle_{k}$ therefore.

It thus can happen that $P_{1}$ and $P_{2}$ are on the same side of an event or particle horizon for some $k_{1}$, while they are on opposite sides for some other $k_{2}$. We shall find various examples of this phenomenon below.

\section{3. $k$-microscopes}

We use the term " $k$-microscope" for an idealized experimental set-up, designed to observe the structure of the quantum spacetime, whose observations are well described by the effective field theory provided by the action $\Gamma_{k}$. The idealization involved here is that the "microscope" is assumed to be characterized by a single scale only so that it is clear which one of the effective field theories $\left\{\Gamma_{k}, 0 \leq k<\infty\right\}$ is to be used for its description. This microscope "sees" a metric $\left\langle g_{\mu \nu}\right\rangle_{k}$ solving the effective field equations of the corresponding action functional $\Gamma_{k}$.

What is the proper resolution $\ell \equiv \ell(k)$ of such a microscope? Or, equivalenty: What is the coarse graining length scale $\ell(k)$ over which the metric is "averaged" when observed with the $k$-microscope? The answer is in general complicated, it 
depends on the details of the experimental set-up.

Here we will use a simple but universal mathematical model of a microscope [25, 44], closely related to the very construction of the effective average action. The input data is the set of metrics $\left\{\left\langle g_{\mu \nu}\right\rangle_{k}\right\}$. The idea is to deduce the relation $\ell=\ell(k)$ from the spectral properties of the scale dependent Laplacian $\boldsymbol{\Delta}_{k} \equiv D^{2}\left(\left\langle g_{\mu \nu}\right\rangle_{k}\right)$ built with the solution of the effective field equation. For every fixed value of $k$, one solves the eigenvalue problem of $-\boldsymbol{\Delta}_{k}$ and studies the properties of the eigenfunctions whose eigenvalue is $k^{2}$, or nearest to $k^{2}$ in the case of a discrete spectrum. We refer to an eigenmode of $-\boldsymbol{\Delta}_{k}$ whose eigenvalue is (approximately) the square of the cutoff $k$ as a "cutoff mode" (COM) and denote the set of all COMs by COM $(k)$.

If we ignore the $k$-dependence of $\boldsymbol{\Delta}_{k}$ for a moment (as it would be appropriate for matter theories in flat space) the COMs are, for a sharp cutoff, precisely the last modes integrated out when lowering the cutoff, since the suppression term in the path integral cuts out all $h_{\mu \nu}$-modes with eigenvalue smaller than $k^{2}$. For a nongauge theory in flat space the coarse graining (averaging) of fields is a well defined procedure, based upon ordinary Fourier analysis. In this case the length $\ell$ is roughly the wave length of the COMs.

This observation motivates the following definition of $\ell$ in quantum gravity. We determine the COMs of $-\boldsymbol{\Delta}_{k}$, analyze how fast these eigenfunctions vary on spacetime, and read off a typical coordinate distance $\Delta x^{\mu}$ characterizing the scale on which they vary. For an oscillatory COM, for example, $\Delta x$ would correspond to an oscillation period. Finally we use the metric $\left\langle g_{\mu \nu}\right\rangle_{k}$ itself in order to convert $\Delta x^{\mu}$ to a proper length. This proper length, by definition, is $\ell$. Repeating the above steps for all values of $k$, we end up with a function $\ell=\ell(k)$. In general one will find that $\ell$ depends on the position on the manifold as well as on the direction of $\Delta x^{\mu}$.

In the following $\ell$ will always denote the intrinsic length scale of the COMs obtained from the above model for a " $k$-microscope". Our experience with theories in flat spacetime suggests that the COM scale $\ell$ is a plausible candidate for a physically sensible resolution function $\ell=\ell(k)$, but there might also be others, depending on 
the experimental setup one has in mind.

As long as the physical radius of curvature, measured with $\left\langle g_{\mu \nu}\right\rangle_{k}$, is much larger than $1 / k$, one can in general use a WKB approximation of the mode functions to show that $\ell(k)$ is roughly given by the classical result $\pi / k$. This $\ell(k)$ is a proper length measured with $\left\langle g_{\mu \nu}\right\rangle_{k}$. The coordinate distance $\Delta x$ from which $\ell$ was obtained (and the proper length obtained when this $\Delta x$ is "measured" with a fixed macroscopic metric $\left\langle g_{\mu \nu}\right\rangle_{k_{\text {lab }}}$ ) may depend on $k$ in a completely different way. In ref. [44] we showed that, in the case of a Euclidean four-sphere, there is a minimal coordinate distance $\Delta x$ with the property that a $k$ exists so that $\Delta x$ can be resolved by the corresponding cutoff modes. This is true although there is no lower bound on $\ell(k)$, which runs $\propto 1 / k$ all the way towards $k \rightarrow \infty$.

In the presence of strong curvature, $\ell(k)$ may deviate substantially from $\pi / k$. It is then in general necessary to write $\ell(k, x, n)$ to account for the dependence on position and direction. We specify the direction by a unit vector $n^{\mu}$.

The $n^{\mu}$-dependence is particularly important if the signature of the metric is Lorentzian. Because of the possible compensation of timelike and spacelike oscillations, one could then have arbitrarily small $\ell$ for arbitrarily small $k$. In flat Minkowski space, say, a wave $\sim e^{i(\vec{k} \vec{x}-|\vec{k}| t)}$ has $k^{2}=0$ but the wavelength $2 \pi /|\vec{k}|$ can be arbitrarily small. A working procedure to determine $\ell(k, x, n)$ for a given direction $n^{\mu}$ would be to define it from the COMs which vary as little as possible in any direction orthogonal to $n$.

\section{The Schwarzschild-de Sitter family}

In this section we illustrate several of the points discussed above by means of explicit examples. We consider pure Lorentzian gravity in the Einstein-Hilbert approximation. The family of effective field equations is given by eq. (3) then.

\subsection{Running metric for a generic state}

Let us find the most general solution to eqs. (3) with $\Lambda(k)>0$ subject to the 
symmetry constraint that $\left\langle g_{\mu \nu}\right\rangle_{k}$ is spherically symmetric (isotropic) and stationary on all scales. On the constant-time surfaces we use polar coordinates $r, \theta, \phi$, and we define the time coordinate such that $\partial / \partial t$ is the Killing vector related to stationarity.

Applying the familiar textbook arguments [45] at each value of $k$ we see that in these coordinates the most general static isotropic line element $\left\langle d s^{2}\right\rangle_{k} \equiv\left\langle g_{\mu \nu}\right\rangle_{k} d x^{\mu} d x^{\nu}$ is given by

$$
\begin{aligned}
\left\langle d s^{2}\right\rangle_{k}=- & F(r ; k) d t^{2}+2 r E(r ; k) d t d r+r^{2} D(r ; k) d r^{2} \\
+ & C(r ; k)\left[d r^{2}+r^{2} d \theta^{2}+r^{2} \sin ^{2} \theta d \phi^{2}\right] .
\end{aligned}
$$

It contains 4 free functions, $C, D, E$ and $F$, which depend on the coordinate $r$ and the parameter $k$.

Let us first recall the situation in classical gravity where the 4 functions are $k$-independent. There one can perform a change of coordinates which reduces the number of free functions to 2 . If one introduces as new coordinates

$$
r^{\prime}=r C(r)^{1 / 2}, \quad t^{\prime}=t+T(r)
$$

with $T(r)=-\int d r r E(r) / F(r)$, then the line element can be brought to the "standard form"

$$
d s^{2}=-B\left(r^{\prime}\right) d t^{2}+A\left(r^{\prime}\right) d r^{\prime 2}+r^{\prime 2}\left[d \theta^{2}+\sin ^{2} \theta d \phi^{2}\right] .
$$

The two new coefficient functions $A$ and $B$ can be expressed in terms of the original ones, see ref. [45].

In quantum gravity where the metric coefficients depend on $k$ an analogous reduction from 4 to 2 free functions is not possible. The reason is that in this case the change of coordinates (15) would involve $k$-dependent functions $C(r ; k)$ and $T(r ; k)$ but, as we discussed in section 2.3, the group of gauge transformations consists of $k$-independent diffeomorphisms only. We would like the coordinates $\left(t_{1}, r_{1}, \theta_{1}, \phi_{1}\right)$ to belong to the same physical point $P_{1}$ for all values of $k$, and therefore only $k$ independent coordinate transformations are possible. We may use the freedom to perform $k$-independent transformations in order to transform $\left\langle d s^{2}\right\rangle_{k}$ to the standard 
form (16) at one particular value of $k$ at most. The gauge transformations are "used up" then, and on all other scales $k^{\prime} \neq k$ one still needs 4 functions $C, D, E$ and $F$ to parametrize the most general static isotropic metric.

The parametrization (14) is now used as an ansatz for solving the familiy of field equations. Inserting (14) into (3) one obtains a coupled system of ordinary differential equations, involving $C, D, E$ and $F$, and its derivatives with respect to $r$. We shall not write down these complicated equations here. Suffice it to say that the equations belonging to different $k$-values are completely decoupled and can be solved for each $k$ separately. But at fixed $k$ the situation is the same as in classical gravity where the field equations determine only two functions, after the other two have been fixed by an appropriate choice of coordinates.

Here we see very explicitly that the field equations cannot fix $\left\langle g_{\mu \nu}\right\rangle_{k}$ completely. As we discussed already, they determine $\left\langle g_{\mu \nu}\right\rangle_{k}$ up to a $k$-dependent diffeomorphism. But since the group of gauge transformations consists of $k$-independent diffeomorphisms only, the family $\left\{\left\langle g_{\mu \nu}\right\rangle_{k} ; 0 \leq k<\infty\right\}$ encodes additional physical information which is "known" to the state $|\Psi\rangle$ only, but not to the effective field equations.

\subsection{A special class of states}

The previous subsection can be summarized by saying that in the static isotropic case the running metric is parametrized by 4 functions of $r$ and $k$. The effective field equations allow us to express 2 of them in terms of the other 2 ; the latter can be found only from $|\Psi\rangle$ or the corresponding path integral directly.

In order to illustrate another point we shall now assume that there exists a class of states $|\Psi\rangle$ for which, for any $k$, the metric assumes the more special form

$$
\left\langle d s^{2}\right\rangle_{k}=-f(r ; k) d t^{2}+\frac{d r^{2}}{g(r ; k)}+r^{2} d \theta^{2}+r^{2} \sin ^{2} \theta d \phi^{2} .
$$

We emphasize that (15) represents an assumption, there is no guarantee that a state with this property exists. Taking the more restricted structure (17) for granted, the effective field equations determine the functions $f$ and $g$ almost completely. Inserting 
(17) into (3) one infers that the most general solution has $g(r ; k)=f(r ; k)$ and

$$
f(r ; k)=1-\frac{2 m(k)}{r}-\frac{\Lambda(k)}{3} r^{2} .
$$

Obviously, for every $k$, (17) with (18) is a Schwarzschild-de Sitter metric for the cosmological constant $\Lambda(k)$. Here $m(k)$ is a constant of integration which may be chosen differently at different scales. While $\Lambda(k)$ is dictated by the RG equation, we cannot deduce $m(k)$ from the effective field equations.

This is an example of the second source of ambiguities mentioned in subsection 2.3: Constants of integration which are just numbers in classical gravity become functions of $k$ in quantum gravity. This type of ambiguity, too, can be resolved only by analyzing the state $|\Psi\rangle$ directly.

As in classical gravity one might interpret $m(k) \equiv G(k) M(k)$ as the product of the running Newton constant and a running black hole mass $M(k)$. From the point of view of the effective field equations this seems a bit artificial, though, since in vacuo they do not contain $G(k)$.

The function $f(r ; k)$ is positive only in a finite portion of spacetime. This region is sandwiched between two horizons at which $f(r ; k)=0$ : the black hole event horizon at $r=r_{\mathrm{e}}(k)$ and the cosmological horizon at $r=r_{\mathrm{c}}(k)$. As long as $m(k)$ and $\Lambda(k)$ are both sufficiently small, we have $r_{\mathrm{e}}(k) \ll r_{\mathrm{c}}(k)$, and their values are approximately

$$
r_{\mathrm{e}}(k) \approx 2 m(k), \quad r_{\mathrm{c}}(k) \approx \sqrt{\frac{3}{\Lambda(k)}} .
$$

In order to get a first understanding of the scenarios which are in principle possible for the "zooming" into the QEG spacetime we consider two special examples for the function $m(k)$. We assume that there exist states $|\Psi\rangle$ giving rise to this particular form of $m(k)$, but strictly speaking there is no guarantee for their existence. However, the first example is well motivated both by explicit perturbative and RG computations which establish the postulated behavior in a certain regime at least. 


\section{(a) $m(k)$ decreases with growing $k$.}

We assume that, in a certain interval of $k$-values, (i) the function $m(k)$ decreases monotonically with increasing $k$, and (ii) the cosmological constant $\Lambda(k)$ is small there so that $r_{\mathrm{c}}(k) \gg r_{\mathrm{e}}(k)$. The second assumption means that we are essentially looking at a Schwarzschild black hole for which the cosmological horizon plays no important role.

Writing $m(k) \equiv M G(k)$ with $M=$ const we see that the assumption (i) amounts to a running $G(k)$ which decreases with increasing $k$. This is exactly the "antiscreening" behavior implied by the RG equations [1]. In fact, in refs. 29] the RG-improved Schwarzschild metric was obtained by taking the running of Newton's constant into account, and contact was made with the quantum corrected Newtonian potential calculated perturbatively within the effective field theory approach to gravity [46, 47]. These explicit analyses indicate that there should indeed exist states with the properties assumed above.

Since $r_{\mathrm{e}}(k) \approx 2 m(k)$, a decreasing $m(k)$ implies that the event horizon moves inward as we increase the scale $k$. As a result, there exist (space) points $Q$ which are inside the horizon according to the metric $\left\langle g_{\mu \nu}\right\rangle_{k_{1}}$, but are outside when one uses $\left\langle g_{\mu \nu}\right\rangle_{k_{2}}$ with $k_{2}>k_{1}$ instead. Let us position some observer at a point $Q^{\prime}$ with $r_{Q^{\prime}}>r_{\mathrm{e}}\left(k_{1}\right)$. Then this observer can receive signals from $Q$ according to the causal structure pertaining to the scale $k_{2}$, but not to the one for $k_{1}$. This is an example of "scale-dependent causality".

While seemingly paradoxical at first sight, its interpretation should be clear from the discussion above: In the quantum regime, the transmission of a signal can no longer be modeled by a (massless) test particle, but rather the backreaction onto the metric of the complete physical system consisting of a transmitter, the signal per se, and a receiver has to be taken into account. In the average action approach this is done, to leading order, by changing the relevant scale $k$.

In describing the transmission process by the set of metrics (17) we made the tacit assumption that it preserves the original symmetries of the spacetime without 
the transmission apparatus. This might be an oversimplification when it comes to describing realistic physical experiments, but it does not affect our general conclusion that causality is a resolution dependent notion, in principle.

(b) $m(k)$ is constant.

Next we assume a state for which the function $m(k) \equiv m_{0}$ is constant and take $G(k)$ and $\Lambda(k)$ to follow a Type IIIa trajectory. Both horizons are now unaffected for $k \lesssim k_{T}$, where $k_{T}$ is the turning point of the trajectory, as described in the Introduction. For $k$ well above $k_{T}, \Lambda(k)$ grows $\propto k^{4}$. This implies that the radius of the cosmological horizon gets smaller as $k$ increases: $r_{\mathrm{c}}(k) \sim k^{-2}$. (This is analogous to the $k^{-2}$-shrinking of the sphere in [44].) At the same time, the inner horizon at $r_{\mathrm{e}}(k)$ is driven outwards because of the growing importance of the term $\frac{\Lambda(k)}{3} r^{2}$ in $f(r ; k)$. Finally, at $k=k_{\mathrm{m}}$, the two horizons merge when $\Lambda(k)$ reaches the value

$$
\Lambda\left(k=k_{\mathrm{m}}\right)=\frac{1}{9 m_{0}^{2}}
$$

at the position

$$
r_{\mathrm{e}}\left(k_{\mathrm{m}}\right)=r_{\mathrm{c}}\left(k_{\mathrm{m}}\right)=3 m_{0}=\frac{1}{\sqrt{\Lambda\left(k_{\mathrm{m}}\right)}} .
$$

The scale $k_{\mathrm{m}}$ where the horizons merge can be in the range where $\Lambda(k) \sim k^{4}$ or in the fixed point regime where $\Lambda(k) \sim k^{2}$, depending on the value of $m_{0}$. For $k>k_{\mathrm{m}}$, $f(r)$ is negative everywhere, and as a result the causal structure of spacetime on these scales is completely different from that at small $k$.

\section{Robertson-Walker families}

Next we analyze the effective field equations imposing a different symmetry requirement. We assume that, for any value of $k$, the effective spacetime is foliated by spacelike hypersurfaces which are homogeneous and isotropic. Specializing further for flat hypersurfaces, the most general metric consistent with these requirements is of the form

$$
\left\langle d s^{2}\right\rangle_{k} \equiv\left\langle g_{\mu \nu}\right\rangle_{k} d x^{\mu} d x^{\nu}=-b^{2}(t ; k) d t^{2}+a^{2}(t ; k) \delta_{i j} d x^{i} d x^{j}
$$


It contains two free functions $a$ and $b$ of the time coordinate $t$. If it were not for their parametric $k$-dependence, one could redefine the time variable $\left(t \rightarrow t^{\prime}=\int b d t\right)$ in order to achieve $b=1$. This would lead us to the standard form of the RobertsonWalker line element as it is usually used in cosmology. However, as the group of gauge transformations consists of $k$-independent diffeomorphisms only, $b=1$ can be achieved for one value of $k$ at most, but not for all. Therefore, if the symmetry requirement is the only subsidiary condition constraining the form of $\left\langle g_{\mu \nu}\right\rangle_{k}$, the most general form of the running metric contains two free functions of $t$ and $k$.

For generic functions $a$ and $b$ the metric (22) admits 6 Killing vectors $K_{a}^{\mu} \partial_{\mu}$ related to homogeneity and isotropy. They are $k$-independent since they just translate and rotate the spatial cartesian coordinates $x^{i}$ in the usual way; these transformations do not involve the $k$-dependent functions $a$ and $b$ as the $x^{i}$ 's do not get mixed with $t$.

\subsection{Vacuum solutions}

Again employing the Einstein-Hilbert truncation, we assume that no matter is present so that the effective Einstein equations assume the form (3). Inserting the ansatz (22) we obtain a single differential equation for $a$ and $b 3$ :

$$
3\left(\frac{\dot{a}(t ; k)}{a(t ; k)}\right)^{2}=\Lambda(k) b^{2}(t ; k) .
$$

Here we encounter another example of the phenomenon that the effective field equations cannot fully determine the physical contents of the running metrics because they are invariant under $k$-dependent diffeomorphisms while physics is invariant under $k$-independent ones only. The single equation (23) does not contain enough information to determine both $a(t ; k)$ and $b(t ; k)$ after fixing initial conditions. The reason is clear: By a $k$-dependent change of the time coordinate we can transform $b$ into any function we like, including $b \equiv 1$, if we use the new time

$$
t^{\prime}(t ; k)=\int^{t} b(\bar{t} ; k) d \bar{t}
$$

\footnotetext{
${ }^{3}$ Here and in the following a dot denotes a derivative with respect to $t$.
} 
But this transformation is forbidden in the present context. The new time coordinate depends on the old one in a scale dependent way which destroys the required $k$ independent one-to-one correspondence between coordinates and physical points.

The allowed transformations are, however, sufficient to achieve $b=1$ at a single scale, $k=k_{0}$ say. Fixing the ( $k$-independent!) coordinate system in this way we can then use (23) to determine $a$ at $k_{0}$ uniquely (up to initial conditions). The result reads

$$
a\left(t ; k_{0}\right)=\exp \left[ \pm \sqrt{\frac{\Lambda\left(k_{0}\right)}{3}}\left(t-t_{1}\right)\right], \quad b\left(t ; k_{0}\right)=1
$$

where $t_{1}$ is an integration constant. Without further information which must come from the state directly it is impossible to determine $a(t ; k)$ and $b(t ; k)$ for $k \neq k_{0}$.

The metric (22) at $k_{0}$, with the functions (25) describes a patch of de Sitter space. As a result, $\left\langle g_{\mu \nu}\right\rangle_{k_{0}}$ has more symmetries than those built into the ansatz. It admits 10 rather than just 6 Killing vectors.

At $k \neq k_{0}$ the state $|\Psi\rangle$ dictates a certain function $b(t ; k) \neq 1$. Nevertheless, the metric $\left\langle g_{\mu \nu}\right\rangle_{k}$ still admits 10 Killing vectors. The reason is that the condition $\mathcal{L}_{K}\left\langle g_{\mu \nu}\right\rangle_{k}=0$ is covariant under $k$-dependent diffeomorphisms. As a consequence, the (non-)existence of Killing vectors cannot depend on whether we use $t$ or $t^{\prime}(k)$ as a time coordinate. Hence $\left\langle g_{\mu \nu}\right\rangle_{k}$ given by (22) with functions $a$ and $b$ constrained by the differential equation (23) but arbitrary otherwise is maximally symmetric for any value of $k$.

The 6 Killing vector fields related to homogeneity and isotropy and the 4 additional ones responsible for the enlarged symmetry are not on an equal footing, though. While the former are $k$-independent, the latter may depend on the scale. The coordinate transformations generated by the former do not mix the $x^{i}$ s with $t$, but the latter do. As a result, the components $K_{a}^{\mu}(x ; k)$ of the 4 additional Killing vectors depend on $k$ explicitly since they "feel" the $k$-dependent coefficient functions $a$ and $b$ in (22). According to the discussion in section 2.4 this implies that the original 6 Killing vectors can be regarded transformations on the manifold $\mathcal{S}$ of physical events, but not necessarily the 4 new ones. We observe a kind of symmetry 
breaking here; it represents an "anomaly" in the sense that it is caused by quantum effects.

This anomaly occurs already if there are states with $b(t ; k)=1$ for all $t$ and $k$. The familiar metric

$$
\left\langle d s^{2}\right\rangle_{k}=-d t^{2}+a(t ; k)^{2} d x^{i} d x^{i}
$$

with the scale factor

$$
a(t ; k)=A \exp \left[\sqrt{\frac{\Lambda(k)}{3}} t\right]
$$

is well known to be maximally symmetric, but even with a $k$-independent constant of integration, $A$, some of the Killing vectors are unavoidably scale dependent because the cosmological constant is.

The cosmology given by (27) has a running Hubble parameter $H(k)=\sqrt{\Lambda(k) / 3}$. Since $\Lambda$ is a monotonically increasing function of $k$, so is $H(k)$. As a result, a highresolution microscope will see the universe expand faster than one with a poorer resolving power. In the fixed point regime where $\Lambda(k) \propto \lambda_{*} k^{2}$, say, we have

$$
a(t ; k)=A \exp \left[\sqrt{\frac{\lambda_{*}}{3}} k t\right]
$$

with $H(k)$ directly proportional to $k$.

It is natural to search for a state $|\Psi\rangle$ in which the full de Sitter symmetries are $k$-independently realized. Only such a state, with $10 k$-independent Killing vector fields, would we call a Quantum de Sitter space, since only then spacetime is maximally symmetric as a manifold $\mathcal{S}$ of physical events. What are the conditions $a(k ; t)$ and $b(t ; k)$ must obey in this case?

The $k$-dependence of the functions $a$ and $b$ must reflect the maximal symmetry of the quantum space time. They have to grow or shrink in the same way as functions of $k$, and this growing or shrinking has to be independent of the position. Taking again $k_{0}$ as a reference scale, these requirements imply

$$
\frac{a(t ; k)}{a\left(t, k_{0}\right)}=\frac{b(t ; k)}{b\left(t ; k_{0}\right)}=c(k),
$$


where $c(k)$ is a function of $k$ only.

To see the necessity of eq. (29) for the full symmetries to hold $k$-independently, we note that maximally symmetric (classical) spacetimes have the following property: Let $u$ be a vector in the tangent space of a point $x$ and $v$ a vector in the tangent space of a point $y$. If $u$ and $v$ have the same length, then there is always an isometry transformation which maps $x \mapsto y$ and $u \mapsto v$ (up to a time reversal). In the quantum case, if all isometries are required $k$-independent, we therefore must demand that if $u$ and $v$ have the same length at some scale $k_{0}$, they automatically have the same length at all scales $k$, since they are always linked by the same isometry transformation. This implies that they obey

$$
\left\langle g_{\mu \nu}(x)\right\rangle_{k} u^{\mu} u^{\nu}=\left\langle g_{\mu \nu}(y)\right\rangle_{k} v^{\mu} v^{\nu}
$$

for all $k$. Since $u$ and $v$ generically point into different directions (e.g. if they are related by an isometry which is a combination of translations, rotations and boosts) this is only possible when all components of the metric have the same dependence on $k$, and when this $k$-dependence is independent of the position. Otherwise $u$ and $v$ would shrink or grow differently when $k$ is changed, and they would be no longer of equal length. This proves eq. (29).

Using (29) in the field equation (23), we see that the only solution for $c(k)$ is

$$
c(k)=\sqrt{\frac{\Lambda\left(k_{0}\right)}{\Lambda(k)}} .
$$

This $k$-dependence of the metric is completely identical to what we found for the four-sphere in ref. [44]. In combination with eq. (25) we obtain the solution for the metrics of quantum de Sitter space:

$$
a(t ; k)=\sqrt{\frac{\Lambda\left(k_{0}\right)}{\Lambda(k)}} \exp \left[ \pm \sqrt{\frac{\Lambda\left(k_{0}\right)}{3}}\left(t-t_{1}\right)\right], \quad b(t ; k)=\sqrt{\frac{\Lambda\left(k_{0}\right)}{\Lambda(k)}} .
$$

We shall return to this result in section 6.2. 


\subsection{Cosmological solutions with matter}

Next we add a matter piece $\Gamma_{k}^{\mathrm{M}}$ to the Einstein-Hilbert ansatz for the average action. The effective field equations assume the form (4) then where the scale dependent energy momentum tensor $\left\langle T^{\mu \nu}\right\rangle_{k}$ is given by (5). We impose the symmetry constraint of homogeneity and isotropy again and consider the case of flat $t=$ const slices. In the adapted $\left(t, x^{i}\right)$-system of coordinates, eq. (22) is the most general metric then and, for symmetry reasons, $\left\langle T_{\mu}{ }^{\nu}\right\rangle_{k} \equiv\left\langle g_{\mu \rho}\right\rangle_{k}\left\langle T^{\rho \nu}\right\rangle_{k}$ has the structure

$$
\left\langle T_{\mu}{ }^{\nu}\right\rangle_{k}=\operatorname{diag}[-\rho, p, p, p]
$$

with functions $\rho \equiv \rho(t ; k)$ and $p \equiv p(t ; k)$. If we insert (33) and the metric ansatz (22) into the effective Einstein equations (4) we obtain two independent differential equations. We take them to be the modified Friedmann equation

$$
3\left(\frac{\dot{a}(t ; k)}{a(t ; k)}\right)^{2}=[8 \pi G(k) \rho(t ; k)+\Lambda(k)] b^{2}(t ; k)
$$

and the continuity equation

$$
\dot{\rho}(t ; k)+3 \frac{\dot{a}(t ; k)}{a(t ; k)}[\rho(t ; k)+p(t ; k)]=0
$$

which corresponds to (17). These equations are to be supplemented by the matter equation of motion or, in a hydrodynamical description of the matter system, by an equation of state $p=p(\rho ; k)$.

\subsubsection{Solutions with $k$-independent causality by constant Weyl rescaling}

In absence of matter there exists a simple general method for generating solutions $\left\langle g_{\mu \nu}\right\rangle_{k}$ if an initial solution $\left\langle g_{\mu \nu}\right\rangle_{k_{0}}$ at some reference scale $k_{0}$ is known: it is enough to multiply the initial metric by an $x$-independent conformal factor [25]. In fact,

$$
\left\langle g_{\mu \nu}(x)\right\rangle_{k}=\frac{\Lambda\left(k_{0}\right)}{\Lambda(k)}\left\langle g_{\mu \nu}(x)\right\rangle_{k_{0}}
$$

is a solution to (3) for any $k$ if it is at $k_{0}$ (excluding, as always, topology/symmetry changes in the $k$-interval considered). An example is the solution (32). Clearly the 
family (36) has the same light cone structure at any $k$ and thus provides an example of scale independent causality.

As we are going to argue that scale independent causality is more the exception than the rule it is important to understand that in the presence of matter constant Weyl transforms can be a solution only under highly non-generic and "unnatural" conditions.

For the metric ansatz (22), eq. (36) is equivalent to

$$
a(t ; k)=\sqrt{\frac{\Lambda\left(k_{0}\right)}{\Lambda(k)}} a\left(t ; k_{0}\right), \quad b(t ; k)=\sqrt{\frac{\Lambda\left(k_{0}\right)}{\Lambda(k)}} b\left(t ; k_{0}\right) .
$$

It is easy to verify that (37) is a solution to (34) and (35) provided the energy density and the pressure scale as follows:

$$
\begin{aligned}
& \rho(t ; k)=\frac{\Lambda(k)}{G(k)}\left(\frac{\Lambda\left(k_{0}\right)}{G\left(k_{0}\right)}\right)^{-1} \rho\left(t ; k_{0}\right), \\
& p(t ; k)=\frac{\Lambda(k)}{G(k)}\left(\frac{\Lambda\left(k_{0}\right)}{G\left(k_{0}\right)}\right)^{-1} p\left(t ; k_{0}\right)
\end{aligned}
$$

If (38) happens to be satisfied we indeed have found a solution with $k$-independent causality. However, the $k$-dependence of $\rho$ and $p$ is dictated independently by the RG flow of $\Gamma_{k}^{\mathrm{M}}$ so that in general there is no reason for (38) to hold. Note in particular that (38) implies a very special "equation of state"

$$
p(t ; k)=w(t) \rho(t ; k)
$$

where

$$
w(t) \equiv p(t ; k) / \rho(t ; k)
$$

depends on time but not on $k$. The factors on the RHS of eqs. (38) have an interesting interpretation. Since

$$
\rho_{\Lambda}(k) \equiv \frac{\Lambda(k)}{8 \pi G(k)}
$$

is the scale dependent (but $t$-independent!) vacuum energy density due to the cosmological constant we may write $\rho$ and $p$ in a form where its $t$ - and $k$-dependencies 
factorize:

$$
\rho(t ; k)=\frac{\rho_{\Lambda}(k)}{\rho_{\Lambda}\left(k_{0}\right)} \rho\left(t ; k_{0}\right)
$$

and likewise for $p$. The above solution with $k$-independent causality exists if, and only if, the matter energy density, at any time, scales with $k$ in the same way as the vacuum energy density.

These remarks indicate that the relations (38) are highly constraining. We illustrate this point for a single scalar field $\chi$ and the "local potential approximation"

$$
\Gamma_{k}^{\mathrm{M}}[g, \chi]=-\int d^{4} x \sqrt{-g}\left(\frac{1}{2}\left(D_{\mu} \chi\right)^{2}+V_{k}(\chi)\right) .
$$

Then, for a spatially homogeneous solution $\langle\chi(t)\rangle_{k}$,

$$
\begin{aligned}
& \rho(t ; k)=\frac{1}{2 b^{2}}\left(\frac{d}{d t}\langle\chi(t)\rangle_{k}\right)^{2}+V_{k}\left(\langle\chi(t)\rangle_{k}\right), \\
& p(t ; k)=\frac{1}{2 b^{2}}\left(\frac{d}{d t}\langle\chi(t)\rangle_{k}\right)^{2}-V_{k}\left(\langle\chi(t)\rangle_{k}\right)
\end{aligned}
$$

Here $\rho$ and $p$ have both an explicit $k$-dependence via the RG running of the effective average potential $V_{k}$ and an implicit one via the solution $\left(\langle g\rangle_{k},\langle\chi\rangle_{k}\right)$. It is clear that for a generic RG trajectory $\left\{G(k), \Lambda(k), V_{k}(\cdot)\right\}$ and generic solution to the resulting $t$-dynamics eqs. (44) will not comply with (38).

One might try to search for solutions with $k$-independent causality by allowing the metrics at different scales to be related by a position-dependent conformal factor:

$$
\left\langle g_{\mu \nu}\right\rangle_{k}=C\left(x ; k, k_{0}\right)\left\langle g_{\mu \nu}\right\rangle_{k_{0}}
$$

However, for a generic RG trajectory and solution to the field equations the metric will not be of the form (45). The reason is clear: in the exact theory the RG trajectory amounts to infinitely many running couplings such as $G(k), \Lambda(k)$, or the function $V_{k}(\cdot)$ which by itself contains already infinitely many couplings. All of these couplings get changed when we switch to another trajectory. At the level of solutions, this change cannot in general be absorbed by a change of the single function $C\left(x ; k, k_{0}\right)$. Therefore, unless one is dealing with a highly symmetric theory or performs an extreme finetuning of initial conditions, a generic solution for the running 
metric will not be of the type (45). As a result, the causal structure will depend on $k$ then.

\subsubsection{Cosmologies with $k$-dependent causality}

In order to illustrate the case of scale dependent causal structures we assume in this section that there exists a class of special states with

$$
b(t ; k)=1 \quad \text { for all } t \text { and } k \text {. }
$$

We employ a hydrodynamical description of the matter system and take the equation of state to be $p=\rho / 3$, corresponding to a traceless energy momentum tensor. Hence the metric reads $\left\langle d s^{2}\right\rangle_{k}=-d t^{2}+a^{2}(t ; k) d \mathbf{x}^{2}$, and the Friedmann equation (34) and continuity equation (35) assume their standard form. For an RG trajectory with $\Lambda(k)>0$ in the $k$-interval of interest their general solution reads:

$$
\begin{gathered}
a(t ; k)=\left[\frac{\mathcal{M}(k) G(k)}{2 \Lambda(k)}\left\{\cosh \left[\frac{4}{3} \sqrt{3 \Lambda(k)}\left(t-t_{0}\right)\right]-1\right\}\right]^{1 / 4}, \\
\rho(t ; k)=\frac{\mathcal{M}(k)}{8 \pi a^{4}(t ; k)} .
\end{gathered}
$$

This solution contains two constants of integration, $\mathcal{M}(k)$ and $t_{0}$. The $k$-dependence of $\mathcal{M}(k)$ is not fixed by the Einstein equations. On the other hand, the constant of integration $t_{0}$ cannot depend on $k$, since this would be inconsistent with our definition of an effective QEG spacetime: The range of the time coordinate is the interval $\left(t_{0}, \infty\right)$. The universe starts with a big bang singularity at $t=t_{0}$. If we had $t_{0}\left(k_{1}\right)<t_{0}\left(k_{2}\right)$, the era between $t_{0}\left(k_{1}\right)$ and $t_{0}\left(k_{2}\right)$ would exist when spacetime is probed at the scale $k=k_{1}$ but not at $k=k_{2}$. This would be in contradiction with our assumption that there is the same one-to-one correspondence between coordinates and events for all $k$. Thus $t_{0}$ must be independent of $k$, and we may set $t_{0}=0$ by readjusting the time axis.

Let us consider the fixed point regime as an example. Every QEG trajectory 4

\footnotetext{
${ }^{4}$ The quantum fluctuations of the matter fields influence the RG flow. The NGFP is known to persist for a broad class of matter systems, however [9].
} 
starts near the NGFP $\left(g_{*}, \lambda_{*}\right)$ where the dimensionful gravitational parameters behave as

$$
G(k)=g_{*} / k^{2}, \quad \Lambda(k)=\lambda_{*} k^{2} .
$$

With this $\mathrm{RG}$ running, valid for $k \gtrsim m_{\mathrm{pl}}$, eq. (47) becomes

$$
a(t ; k)=\frac{1}{k}\left[\frac{g_{*} \mathcal{M}(k)}{2 \lambda_{*}}\right]^{1 / 4}\left\{\cosh \left[\frac{4}{3} \sqrt{3 \lambda_{*}} k t\right]-1\right\}^{1 / 4} .
$$

It is instructive to analyze (50) in the regimes where $k$ is much smaller or larger than the second mass scale in the problem, $1 / t$. We have

$$
\begin{aligned}
& a(t ; k)=\left(\frac{g_{*}}{6}\right)^{1 / 4} \mathcal{M}(k)^{1 / 4} \sqrt{t / k} \text { for } \quad m_{\mathrm{Pl}} \lesssim k \ll 1 / t, \\
& a(t ; k)=\left(\frac{g_{*} \mathcal{M}}{2 \lambda_{*}}\right)^{1 / 4} \frac{1}{k} \exp \left(\sqrt{\lambda_{*} / 3} k t\right) \quad \text { for } \quad k \gg 1 / t .
\end{aligned}
$$

A "microscope" with a comparatively poor resolution, corresponding to a small $k \ll 1 / t$, sees essentially the classical $a \propto \sqrt{t}$ expansion. Since $1 / t \propto H(t)$ here, this microscope focuses on "super-Hubble" structures. On the other extreme, a high resolution microscope with $k \gg 1 / t$ perceives the universe as exponentially inflating. Its Hubble parameter $H=\sqrt{\lambda_{*} / 3} k$ is constant in time but depends on $k$ : the better the resolution of the microscope is, the faster seems the universe to expand. This phenomenon is related to the fractal and self-similar properties of QEG spacetimes discussed earlier [3, 5, 25].

At this point we can make contact with the RG improvement approach. A microscope whose resolution is continuously readjusted so that $k=1 / t$ sees the universe expanding according to

$$
a(t ; k) \propto(\mathcal{M}(1 / t))^{1 / 4} t \quad \text { for } \quad k=1 / t .
$$

For $\mathcal{M}=$ const one recovers precisely the linear expansion $a \propto t$ which was found in [30] by a completely different reasoning.

The causal structure of the spacetime with the scale factors (150) does indeed depend on $k$. This becomes manifest when one investigates possible particle horizons, 
for example. A Robertson-Walker metric implies a particle horizon of coordinate (or comoving) radius

$$
r_{H}(t ; k)=\int_{0}^{t} \frac{d t^{\prime}}{a\left(t^{\prime} ; k\right)}
$$

provided the integral on the RHS of (54) converges at its lower limit. If $r_{H}$ is finite, a fixed event at $r=0$ and time $t$ can be influenced causally only by the events inside a spatial ball with this coordinate radius. Now, since the relationship between coordinates and events is strictly $k$-independent, a scale dependence of $r_{H}(r ; k)$ means that the set of events which can influence the event at $r=0$ and time $t$ is $k$-dependent. For the $a(t ; k)$ of eq. (501) this is indeed seen to happen.

The radius (54) corresponds to the proper distance

$$
d_{H}(t ; k)=a(t ; k) \int_{0}^{t} \frac{d t^{\prime}}{a\left(t^{\prime} ; k\right)}
$$

when lengths are measured with $\left\langle g_{\mu \nu}\right\rangle_{k}$. Note also that with the more general metric (22) eq. (54) gets replaced by

$$
r_{H}(t ; k)=\int_{0}^{t} d t^{\prime} \frac{b\left(t^{\prime} ; k\right)}{a\left(t^{\prime} ; k\right)} .
$$

This indicates again that the second, undetermined metric function can acquire a physical significance when RG effects are taken into account.

More information would probably be available when one investigates what properties a "reasonable" state would have, particularly in the matter sector. In late cosmology, where matter is given as a set of particles with only weakly $k$-dependent masses $m(k)$, the running of $\rho(t ; k)$ essentially boils down to the running of the scale factor. It is reasonable to assume that at any given time $t_{1}$, the number of particles varies only very slightly with $k$, up to very high energies. Then one can use information about the $k$-dependent masses of these particles to relate $\rho\left(t_{1} ; k\right)$ with $a\left(t_{1} ; k\right)$ (the number density of particles is proportional to $\left.a\left(t_{1} ; k\right)^{-3}\right)$, now considered as functions of $k$ only:

$$
\rho\left(t_{1} ; k\right)=m(k) n a\left(t_{1} ; k\right)^{-3},
$$


where $n$ is the number density per unit coordinate volume. This determines the cosmological solutions to a greater extent.

One of the main arguments used for the motivation of inflation is the so-called "horizon problem". The statement is that at the time when the microwace background radiation was emitted, far-separated regions of the universe had very similar properties although they had up to then no time to "communicate" with each other, in the framework of standard cosmology without inflation. We wish to emphasize here the possibility that the horizon problem might be solvable by the $k$-dependent causality structure of spacetime, without inflation. For the high energy processes of the very early universe (e.g. in the Planck era), it is very likely that the most appropriate description is obtained when one uses a $k$-microscope with very large $k$. For such a view on the early universe, it may well be that regions appear causally connected that would be far outside each other's horizons within the classical description, i.e. with $\left\langle g_{\mu \nu}\right\rangle_{k_{\text {lab }}}$. Note in particular that the term "Planck era" refers to the set of spacetime points which are separated from the big bang by less than a Planck time when time is defined via $\left\langle g_{\mu \nu}\right\rangle_{k_{\mathrm{lab}}}$.

A first encouraging result indicating that quantum gravity might solve the horizon problem without inflation was found in [30] in the context of RG improved field equations. Their solution corresponding to the very early universe does not have a particle horizon!

\section{The concept of an intrinsic scale}

We continue to consider homogeneous and isotropic cosmologies described by metrics of the form (22). Let us consider an arbitrary physical structure in the QEG spacetime which has a comoving length $\Delta x$. A typical example would be the wavelength of some perturbation. Then the running metric $\left\langle g_{\mu \nu}\right\rangle_{k}$ associates to the fixed coordinate length $\Delta x$ the running proper length

$$
L(t ; k)=a(t ; k) \Delta x .
$$

What would be a sensible choice for the scale $k$ when one tries to observe the $\Delta x$ - 
object by means of a " $k$-microscope"? Following the discusion of the "intrinsic scale" in ref. [44] a plausible choice seems to be the scale $k \equiv k_{\text {in }}(\Delta x, t)$ at which the resolving power of the microscope, $\ell(k)$, equals precisely the yet to be determined proper length of the object:

$$
L\left(t ; k_{\text {in }}(\Delta x, t)\right)=\ell\left(k_{\text {in }}(\Delta x, t)\right)
$$

If $\ell=\ell(k)$ and the scale factor $a(t ; k)$ are known, this condition yields the following implicit equation for $k_{\text {in }}(\Delta x, t)$ :

$$
a\left(t ; k_{\text {in }}(\Delta x, t)\right) \Delta x=\ell\left(k_{\text {in }}(\Delta x, t)\right) .
$$

If this equation has a unique solution it is natural to define the folowing length $L_{\text {in }}(t)$ as the ( $t$-dependent, but $k$-independent) proper length "intrinsic" to the $\Delta x$-object:

$$
L_{\text {in }}(t) \equiv L\left(t ; k_{\text {in }}(\Delta x, t)\right)=a\left(t ; k_{\text {in }}(\Delta x, t)\right) \Delta x
$$

As in classical cosmology, we may refer to the ratio of the object's proper length and comoving length as a scale factor. However, in the present case this ratio

$$
L_{\text {in }}(t) / \Delta x=a\left(t ; k_{\text {in }}(\Delta x, t)\right) \equiv a_{\text {in }}(t ; \Delta x)
$$

yields a scale factor $a_{\text {in }}(t ; \Delta x)$ which itself depends on $\Delta x$ and, in a sense, is "intrinsic" to the $\Delta x$-object. Stated differently, objects of different comoving size are affected by the cosmological expansion in different ways; each of them has its own scale factor,

$$
L_{\text {in }}(t)=a_{\text {in }}(t ; \Delta x) \Delta x,
$$

and its own $b_{\text {in }}(t ; \Delta x) \equiv b\left(t ; k_{\text {in }}(\Delta x, t)\right)$. Though surprising at first sight, the physical mechanism behind this phenomenon is clear: Objects of different sizes are optimally described by taking the gravitational couplings at different scales, and as a result the effective spacetime they determine is different from object to object.

A similar discussion applies to temporal proper distances. Let us consider two events $P_{1}$ and $P_{2}$ which have identical $x^{i}$-coordinates and $t$-coordinates differing by 
an amount $\Delta t>0$ with respect to the system of coordinates in which (22) is written. According to the metric $\left\langle d s^{2}\right\rangle_{k}$ the proper time elapsed between the events is

$$
T(t ; k)=b(t ; k) \Delta t
$$

As in the spatial case, one can try to adjust $k \equiv k_{\text {in }}(\Delta t, t)$ in such a way that the resulting proper time matches exactly the resolving power:

$$
b\left(t ; k_{\text {in }}(\Delta t, t)\right) \Delta t=\ell\left(k_{\text {in }}(\Delta t, t)\right)
$$

If this equation has a unique solution the natural definition for the proper time distance "intrinsic" to the two events is

$$
T_{\text {in }}(t) \equiv T\left(t ; k_{\text {in }}(\Delta t, t)\right) \Delta t=b\left(t ; k_{\text {in }}(\Delta t, t)\right) \Delta t
$$

It is derived from the explicitly $\Delta t$-dependent Robertson-Walker metric with $a_{\text {in }}(t ; \Delta t) \equiv a\left(t ; k_{\text {in }}(\Delta t, t)\right)$ and $b_{\text {in }}(t ; \Delta t) \equiv b\left(t ; k_{\text {in }}(\Delta t, t)\right)$.

We shall illustrate the points made above employing the COM definition of the resolving power $\ell(k)$ which was explained in section 3. In particular we assume that the WKB approximation is valid so that approximately $\ell(k)=\pi / k$. In this case we have $k_{\text {in }}(\Delta t, t)=\pi / L_{\text {in }}(t)$ which, when inserted into (61), leads to an implicit equation directly for $L_{\text {in }}(t)$ :

$$
L_{\mathrm{in}}(t)=a\left(t ; \pi / L_{\mathrm{in}}(t)\right) \Delta x
$$

For intrinsic proper time intervals we use the same $\ell(k)$, whence

$$
T_{\text {in }}(t)=b\left(t ; \pi / T_{\text {in }}(t)\right) \Delta t
$$

Next we turn to various examples.

\subsection{Example: de Sitter family with $k$-dependent Killing vectors}

Our first example is the de Sitter type family of metrics (26) with (28) which has some of its symmetries implemented in a $k$-dependent way. It is based upon the 
fixed point running $\Lambda(k)=\lambda_{*} k^{2}$. For this family, eq. (67) reads

$$
L_{\text {in }}(t)=A \Delta x \exp \left[\sqrt{\frac{\lambda_{*}}{3}} \frac{\pi t}{L_{\text {in }}(t)}\right] .
$$

This functional equation can be solved in terms of the Lambert $W$-function $5 W_{0}$ :

$$
L_{\text {in }}(t)=\frac{\alpha t}{W(\alpha t / A \Delta x)}
$$

Here $\alpha \equiv \pi \sqrt{\lambda_{*} / 3}$, and the corresponding intrinsic scale factor reads

$$
a_{\text {in }}(t ; \Delta x)=\frac{\alpha t}{\Delta x W(\alpha t / A \Delta x)}
$$

We see that it depends explicitly on the size of the object whose size is measured with the corresponding metric. While it seems absurd from the point of view of classical Riemannian geometry, this phenomenon is very natural from a quantum field theory perspective. The $\Delta x$-dependence of $a_{\text {in }}$ simply reflects the fact that large objects "feel" the value of the gravitational parameters on other scales than small objects do. Since $\Lambda(k) \propto k^{2} \propto 1 / L^{2}$ is the smaller the larger $L$ is, small objects will appear to expand faster than larger ones.

We can display this behavior analytically by specializing for objects which are much smaller than $\alpha t$. If $y \equiv \alpha t / A \Delta x \gg 1$ we may use the following asymptotic expansion of $W_{0}$ for $x \rightarrow \infty$ :

$$
W_{0}(y)=\ln y-\ln \ln y+\frac{\ln \ln y}{\ln y}+\ldots
$$

Retaining only the first term we get approximately

$$
a_{\mathrm{in}}(t ; \Delta x)=\frac{\alpha t}{\Delta x \ln (\alpha t / A \Delta x)} .
$$

Remarkably, apart from a logarithmic correction, $a_{\text {in }}$ is a linear rather than exponential function of time (as it was classically): $a_{\text {in }} \propto t / \Delta x$. This slowing down of

\footnotetext{
${ }^{5}$ By definition [48, the $W$-function satisfies $W(x) \exp [W(x)]=x$, and $W \equiv W_{0}$ denotes its real branch analytic at $x=0$.
} 
the expansion by quantum effects is easy to understand: as the universe expands and $L_{\text {in }}(t)$ becomes larger, the object considered feels an ever decreasing cosmological constant. The combined effect of the exponential expansion and the continuous reduction of $\Lambda(k)$ is the linear expansion (73) .

Furthermore, we can look at objects of different size at a fixed time. Because we have approximately $a_{\text {in }} \propto t / \Delta x$ we see that large objects have indeed a lower expansion rate than small ones.

So far we concentrated on the innermost "core" of the QEG spacetime. When we move "outward", $\Lambda(k)$ leaves the NGFP regime. If it approaches a constant value below some $k$, the spacetime will look like a classical de Sitter space macroscopically. If this constant is zero, it will approach a standard smooth Minkowski space on large distance scales.

It is quite intriguing that even Minkowski space, if we put it under a sufficiently strong microscope, might show a complicated pattern of cosmological constant driven "expansions" which we usually consider in macroscopic cosmology only. One has to be careful in applying this cosmology-type picture, however, since it provides only a local description in the domain accessible by the highly symmetric Robertson-Walker metric. In fact, our simple model for the observation of spacetime by a "microscope" assumes that even in presence of the (back-reacting!) microscope the universe is homogeneous and isotropic. In realistic experiments this will not be the case presumably so that one has to deal with families $\left\langle g_{\mu \nu}\right\rangle_{k}$ with less or no symmetry which are clearly much harder to come by.

\subsection{Example: de Sitter family with $k$-independent Killing vectors}

Our second example is the "anomaly free" de Sitter family with 10 scale independent Killing vectors. As we found in eq. (32), it corresponds to

$$
a(t ; k)=\sqrt{\frac{\Lambda\left(k_{0}\right)}{\Lambda(k)}} \exp \left(H_{0} t\right), \quad b(t ; k)=\sqrt{\frac{\Lambda\left(k_{0}\right)}{\Lambda(k)}},
$$


where $H_{0}=\sqrt{\Lambda\left(k_{0}\right) / 3}$. We analyze this family for a model trajectory of the type

$$
\Lambda(k)=B k^{\omega}, \quad B>0,
$$

with an arbitrary constant exponent $\omega \geq 0$. Important special cases include $\omega=0$ (classical dS), $\omega=2$ (fixed point regime) and $\omega=4$ ( $k^{4}$-regime). Therefore, with the abbreviation $\gamma \equiv \sqrt{B / \Lambda\left(k_{0}\right)}$,

$$
a(t ; k)=\gamma^{-1} k^{-\omega / 2} \exp \left(H_{0} t\right), \quad b(t ; k)=\gamma^{-1} k^{-\omega / 2}
$$

so that the self-consistency condition (67) assumes the form

$$
L_{\text {in }}(t)=\gamma^{-1}\left(\frac{L_{\text {in }}(t)}{\pi}\right)^{\omega / 2} \exp \left(H_{0} t\right) \Delta x .
$$

Its solution is easily found:

$$
L_{\text {in }}(t)=\pi^{\omega /(\omega-2)}\left(\frac{\gamma}{\Delta x}\right)^{2 /(\omega-2)} \exp \left[-\left(\frac{2}{\omega-2}\right) H_{0} t\right] .
$$

The corresponding scale factor reads

$$
a_{\text {in }}(t ; \Delta x)=\beta \exp \left[-\left(\frac{2}{\omega-2}\right) H_{0} t\right] .
$$

where we introduced

$$
\beta \equiv \gamma^{2 /(\omega-2)}\left(\frac{\pi}{\Delta x}\right)^{\omega /(\omega-2)} .
$$

Together with $b_{\text {in }}(t ; \Delta x)=b\left(t ; \pi / L_{\text {in }}(t)\right)$, given explicitly by

$$
b_{\text {in }}(t ; \Delta x)=\beta \exp \left[-\left(\frac{\omega}{\omega-2}\right) H_{0} t\right]
$$

it constitutes the Robertson-Walker metric "intrinsic" to structures of comoving size $\Delta x$ :

$$
\left\langle d s^{2}\right\rangle_{\text {in }} \equiv\left\langle d s^{2}\right\rangle_{k_{\text {in }}}=-b_{\text {in }}^{2}(t ; \Delta x) d t^{2}+a_{\text {in }}^{2}(t ; \Delta x) d \mathbf{x}^{2} .
$$

The line element (82) reads in explicit form

$$
\left\langle d s^{2}\right\rangle_{\text {in }}=\beta^{2} \exp \left[\left(\frac{2 \omega}{\omega-2}\right) H_{0} t\right]\left\{-d t^{2}+\exp \left(2 H_{0} t\right) d \mathbf{x}^{2}\right\} .
$$


As it should be, this equation coincides with $\left\langle d s^{2}\right\rangle_{\text {in }}=\left[\Lambda\left(k_{0}\right) / \Lambda\left(k_{\text {in }}\right)\right]\left\langle d s^{2}\right\rangle_{k_{0}}$. In fact, from (78) and $k_{\text {in }}=\pi / L_{\text {in }}$ we obtain the following expression for the intrinsic scale:

$$
k_{\text {in }}(t ; \Delta x)=\left(\frac{\Delta x}{\pi \gamma}\right)^{2 /(\omega-2)} \exp \left[\left(\frac{2}{\omega-2}\right) H_{0} t\right] .
$$

It is instructive to rewrite it in terms of the proper length $L\left(t ; k_{0}\right)$ measured with $\left\langle d s^{2}\right\rangle_{k_{0}}$ :

$$
k_{\text {in }}(t ; \Delta x)=k_{0}^{\omega /(\omega-2)}\left(L\left(t ; k_{0}\right) / \pi\right)^{2 /(\omega-2)} .
$$

The above results are somewhat surprising and several comments are in order here. For $\omega=0$ we recover the classical results: the scale factor grows as $a_{\text {in }} \propto$ $\exp \left(H_{0} t\right)$ and is independent of $\Delta x, b_{\text {in }}$ is constant, and eq. (85) yields the expected inverse proportionality $k_{\text {in }} \propto 1 / L\left(t ; k_{0}\right)$.

For a fixed $\Delta x$, we are free to bring the "intrinsic" metric into the "true" Robertson-Walker form, i.e. to adjust the time coordinate such that $b_{\text {in }}=1$ at all times. For $\omega \neq 0,2$ we define

$$
\tilde{t} \equiv \int b_{\text {in }}(t ; \Delta x) d t=\beta \frac{2-\omega}{\omega H_{0}}\left(\exp \left[\frac{\omega}{2-\omega} H_{0} t\right]-1\right) .
$$

We fixed the integration constant such that $\tilde{t}(t=0)=0$. Now obviously $b_{\text {in }}(\tilde{t}, \Delta x)=$ 1 , and the scale factor becomes

$$
a_{\text {in }}(\tilde{t} ; \Delta x)=\beta\left[1+\frac{\omega H_{0} \tilde{t}}{(2-\omega) \beta}\right]^{2 / \omega} .
$$

The cosmology with the scale factor (87) is quite remarkable: For $\omega>2$ it describes a contracting rather than expanding universe, even though $a(t ; k) \propto \exp \left(+H_{0} t\right)$ grows for every fixed value of $k$. Moreover, the "intrinsic history" of the object of size $\Delta x$ ends in a "big crunch" singularity at

$$
\tilde{t}=\frac{(\omega-2) \beta}{\omega H_{0}} .
$$

The case $\omega=4$ is particularly relevant since the middle part of most trajectories is well approximated by $\Lambda \propto k^{4}, G=$ const. For the separatrix this " $k^{4}$-regime" 
even extends to $k=0$. For $\omega=4$, the scale factor (87) is

$$
a_{\mathrm{in}}^{\omega=4}(\tilde{t}, \Delta x)=\frac{\gamma \pi^{2}}{(\Delta \mathbf{x})^{2}} \sqrt{1-\frac{2 H_{0}(\Delta \mathbf{x})^{2}}{\gamma \pi^{2}}} \tilde{t} .
$$

Its "big crunch" singularity is not reached actually, since $\Delta x$ at some point leaves the $\omega=4$-regime and enters the fixed point regime with $\omega=2$. Furthermore, eq. (85) becomes

$$
k_{\text {in }}^{\omega=4}(t ; \Delta x)=k_{0}^{2} L\left(t ; k_{0}\right) / \pi,
$$

i.e. rather than inversely, the scale $k_{\text {in }}$ is directly proportional to $L\left(t ; k_{0}\right)$.

These results seem to be counter-intuitive, but they have a natural physics explanation, in fact. The effective time evolution of $a_{\text {in }}$ is given by the equation

$$
\frac{d}{d t} a_{\text {in }}(t ; \Delta x)=\partial_{t} a\left(t ; k_{\text {in }}(t ; \Delta x)\right)+\left.\left(\partial_{t} k_{\text {in }}(t ; \Delta x)\right) \partial_{k} a(t ; k)\right|_{k=k_{\text {in }}(t ; \Delta x)} .
$$

The first term on the right hand side of (91) is the time derivative at fixed $k$ which is dictated by the effective field equations at the fixed scale $k$. In our case it is given by the exponential growth $\propto \exp \left(H_{0} t\right)$. The second term accounts for the rescaling of $a_{\text {in }}$ due to the change of $k$. It is in general cosmologies a property of the particular state considered and is usually only to a small extent determined by the flow equations. In our highly symmetric case it is determined by the factor $\sqrt{\Lambda\left(k_{0}\right) / \Lambda(k)}$. The term $\partial_{t} k_{\text {in }}(t ; \Delta x)$ is itself a function of the LHS, $(d / d t) a_{\text {in }}$, and therefore (91) is an implicit equation. For $\omega>0$, the term $\partial_{k} a(t ; k)$ amounts to a "shrinking" of space with increasing $k$. The effective contraction described by (87) is explained by noting that for $\omega>2$ this shrinking overcomes the exponential growth from the first term, with the result of a decreasing $a_{\text {in }}$.

The explanation for eq. (90) is similar. The shrinking due to the large negative value of $\partial_{k} a(t ; k)$ is so strong that a larger coordinate distance $\Delta x \equiv L\left(t ; k_{0}\right) / a\left(t ; k_{0}\right)$ corresponds to a smaller proper length $L_{\text {in }} \equiv \Delta x a\left(t ; k_{\text {in }}\right)$ and a larger value of $k_{\text {in }}$. This is completely analogous to the shrinking $S^{4}$ discussed in [44]. See also the more detailed discussion there. 
Finally we note that the notion of an "intrinsic length" breaks down directly at the non-Gaussian fixed point, i.e. when $\omega=2$ exactly. This can be already seen from eq. (177). In the fixed point regime, (177) becomes

$$
L_{\mathrm{in}}(t)=\Delta x \frac{L_{\mathrm{in}}(t)}{\pi} \sqrt{\frac{\Lambda\left(k_{0}\right)}{\lambda_{*}}} \exp \left(H_{0} t\right)
$$

$L_{\text {in }}(t)$ appears on both sides linearly and drops out, so the equation cannot be solved. We already met this ill-defined situation in our previous paper [44] in the case of the four-sphere, which had a $k$-dependent radius which was also proportional to $\Lambda(k)^{-1 / 2}$. In the fixed point regime, there is only one value of $\Delta x$ which can be observed with an "appropriate" microscope. Within our parametrization the value is time dependent,

$$
\Delta x=\pi \sqrt{\frac{\lambda_{*}}{\Lambda\left(k_{0}\right)}} \exp \left(-H_{0} t\right) .
$$

When one tries to zoom deeper into this coordinate separation, spacetime locally "shrinks" in a way so that it precisely cancels the effect of zooming.

To explain this, we note that in the fixed point regime $L(t ; k)$ is proportional to $1 / k$. This means that spacetime "shrinks" when we increase the resolution $k$. The length $L_{\text {in }}(t)$ usually arises by finding an "appropriate" value of $k, k=k_{\text {in }}(t ; \Delta x)$, so that $L_{\text {in }}$ fulfills eq. (59). In the example of section 6.1. we found for any $t$ precisely one appropriate value of $k_{\text {in }}$, as one would usually expect. But now, with the relation $L(t ; k) \propto 1 / k$, the correspondence breaks down. For the unique time at which eq. (93) is fulfilled for some given $\Delta x$, every $k$ in the fixed point regime is an appropriate value for $k_{\text {in }}$, and therefore there is no preferred value for $L_{\text {in }}$. At any other time, in contrast, there is no solution for $k_{\text {in }}$ and therefore again no preferred value for $L_{\text {in }}$.

These considerations show that the approximate and somewhat heuristic notion of an intrinsic scale of an object and the spacetime geometry "felt" by this object does not always work as unambiguously as in the example of section 6.1. 
One obtains similar results for intrinsic proper distances in the time direction. The scale is $t$-independent in this case,

$$
k_{\text {in }}(t ; \Delta t)=\left(\frac{\Delta t}{\pi \gamma}\right)^{2 /(\omega-2)},
$$

and the metric coefficients read

$$
\begin{gathered}
a_{\text {in }}(t ; \Delta t)=\gamma^{2 /(\omega-2)}\left(\frac{\pi}{\Delta t}\right)^{\omega /(\omega-2)} \exp \left(H_{0} t\right), \\
b_{\text {in }}(t ; \Delta t)=\gamma^{2 /(\omega-2)}\left(\frac{\pi}{\Delta t}\right)^{\omega /(\omega-2)} .
\end{gathered}
$$

Here $a_{\text {in }}$ has the classical time dependence $\propto \exp \left(H_{0} t\right)$ for any value of $\omega$, and $b_{\text {in }}$ is time independent. The relationship between $\Delta t$ and the intrinsic proper time interval is likewise $t$-independent:

$$
T_{\mathrm{in}}=\pi^{\omega /(\omega-2)}\left(\frac{\gamma}{\Delta t}\right)^{2 /(\omega-2)} .
$$

If $\omega=4$, for example,

$$
T_{\text {in }}^{\omega=4}=\pi^{2} \gamma \frac{1}{\Delta t}
$$

The "shrinking" of spacetime with growing $k$ is again so strong that a larger coordinate interval $\Delta t$ corresponds to a smaller proper intrinsic time $T_{\mathrm{in}}$.

\subsection{Example: Robertson-Walker cosmology with relativistic fluid}

In order to connect our formalism to results obtained in earlier work ("RG improved field equations" [30]), we finally consider a Robertson-Walker spacetime filled by a relativistic fluid. We restrict ourselves to the following situation:

- $b(t ; k)=1$ for all $t$ and $k$.

- The equation of state parameter $w$ is $k$-independently $1 / 3$, so that the solution eq. (47) is valid for every $k$.

- The parameter $\mathcal{M}$ introduced in eq. (47) is $k$-independent.

- We consider the fixed point running of $G$ and $\Lambda$, eq.(49). 
- $k \ll 1 / t$ or $k \gg 1 / t$, so that either eq. (51) or eq. (52) is valid.

The motivation for these specializations (except the last) is that the RG improvement discussed in [30] applies to the same situation. Starting with eq. (51), we have to solve

$$
L_{\text {in }}(t)=\Delta x a\left(t, k=\frac{\pi}{L_{\text {in }}}\right)=\Delta x(\alpha \mathcal{M})^{1 / 4} \sqrt{t L_{\text {in }} / \pi}
$$

(where $\alpha \equiv g_{*} / 6$ ) which yields the linear growth

$$
L_{\text {in }}(t)=(\Delta x)^{2}(\alpha \mathcal{M})^{1 / 2} \frac{t}{\pi}
$$

The condition for the validity of eq. (51), $k_{\text {in }} \ll 1 / t$, implies

$$
t \ll \frac{L_{\text {in }}}{\pi}=\left(\frac{\Delta x}{\pi}\right)^{2}(\alpha \mathcal{M})^{1 / 2} t
$$

or

$$
\Delta x \gg \frac{\pi}{(\alpha \mathcal{M})^{1 / 4}} .
$$

In the universe we live in the parameter $\mathcal{M}$ relevant to the radiation dominated epoch is of the order of magnitude

$$
\mathcal{M}^{1 / 4} \approx 10^{-30} \frac{a_{0}}{\ell_{\mathrm{Pl}}}
$$

where $a_{0}$ is the value of the scale factor today. Assuming that $g_{*}$ is of the order 1 we obtain that the above approximation is valid for objects which have today a size

$$
L_{\text {today }}=\Delta x a_{0} \gg 10^{30} \ell_{\mathrm{Pl}} \approx 10^{-3} \mathrm{~cm} .
$$

The same analysis for eq. (52) amounts to solving the equation

$$
L_{\mathrm{in}}(t)=\Delta x a\left(t, k=\frac{\pi}{L_{\mathrm{in}}}\right)=\Delta x \frac{L_{\mathrm{in}}}{\pi}(\beta \mathcal{M})^{1 / 4} \exp \left(\sqrt{\frac{\lambda_{*}}{3}} \frac{\pi}{L_{\mathrm{in}}} t\right),
$$

where now $\beta \equiv g_{*} / 2 \lambda_{*}$. The solution for $L_{\text {in }}$ is again linear in $t$ :

$$
L_{\mathrm{in}}(t)=\pi t \sqrt{\frac{\lambda_{*}}{3}}\left\{\log \left[\frac{\pi}{\Delta x}\left(\frac{1}{\beta \mathcal{M}}\right)^{1 / 4}\right]\right\}^{-1} .
$$


The condition for the validity of eq. (52),$k_{\text {in }} \gg 1 / t$, implies

$$
t \gg \frac{L_{\text {in }}}{\pi}=t \sqrt{\frac{\lambda_{*}}{3}}\left\{\log \left[\frac{\pi}{\Delta x}\left(\frac{1}{\beta \mathcal{M}}\right)^{1 / 4}\right]\right\}^{-1}
$$

or

$$
\Delta x \ll \frac{\pi}{(\beta \mathcal{M})^{1 / 4}} \exp \left(-\sqrt{\frac{\lambda_{*}}{3}}\right) .
$$

Assuming that $\beta$ and $\exp \sqrt{\lambda_{*} / 3}$ are of order 1 , we get for the case of our universe that the above analysis is valid if

$$
L_{\text {today }}=\Delta x a_{0} \ll 10^{30} \ell_{\mathrm{Pl}} \approx 10^{-3} \mathrm{~cm} \text {. }
$$

In both cases, $k \ll 1 / t$ and $k \gg 1 / t$, we found a linear intrinsic expansion $a_{\text {in }} \propto t$, valid as long as the object is well inside the fixed point regime. In the interpolating transition region $k \approx 1 / t$ we expect a qualitatively similar behavior. A universe with such a time dependence of the scale factor has no particle horizon according to eq. (54). So we have indeed found a case in which the horizon problem does not occur and therefore cannot serve as an argument for inflation. The result $a_{\text {in }} \propto t$ obtained here gives independent support to the linear expansion found by RG improving the field equations [30].

\section{Summary}

In this paper we analyzed various conceptual issues related to a scale dependence of the metric. The discussion is relevant to the asymptotic safety scenario for gravity and, more generally, to the analysis of all phenomena with a strong RG running of the average metric. We described the role of the running effective field equations implied by the average action of QEG and their solutions $\left\{\left\langle g_{\mu \nu}\right\rangle_{k}, 0 \leq k<\infty\right\}$.

The field equations derived from the effective average actions $\left\{\Gamma_{k}\right\}$ allow for infinitely many solutions at each value of $k$. We can only determine from them the set of solutions at any separate value of $k$, but not the evolution $k \mapsto\left\langle g_{\mu \nu}\right\rangle_{k}$ corresponding to a particular quantum state $|\Psi\rangle$. We observed two sources of ambiguities 
which cannot be resolved without knowledge of $|\Psi\rangle$. First, integration constants like the parameter $M$ in the classical Schwarzschild solution become functions of $k$ in the quantum case. Second, simplifications of the metric due to appropriate coordinate transformations can be made for one value of $k$ only. An example was the Robertson-Walker metric $d s^{2}=-b(t ; k)^{2} d t^{2}+a(t ; k)^{2} d \mathbf{x}^{2}$. While $b$ can be set equal to one by a redefinition of the time coordinate in classical gravity, this is possible for only one chosen value of $k$ in the quantum case. We have therefore two functions of $t$ and $k$ instead of one, but the field equations still determine only one of them.

Only for a maximally symmetric spacetime, i.e. de Sitter space, it was possible to determine the evolution $k \mapsto\left\langle g_{\mu \nu}\right\rangle_{k}$ completely from the field equations and special symmetry requirements. We found that the de Sitter metric scales as $\left\langle g_{\mu \nu}\right\rangle_{k} \propto$ $\Lambda(k)^{-1}$.

The scale dependent metric as well as the scale dependent structure of the propagators lead to a scale dependent notion of causality. Outside the classical regime, the position or even existence of horizons generically depend on the field chosen to transmit a signal and on the value of $k$ relevant for the transmission process.

This is particularly interesting for the early universe, since it might surround the necessity of inflation: The particle horizons leading to the so-called horizon problem (which is one of the main arguments for inflation) are the classical horizons which may be irrelevant at typical scales governing processes in the very early universe.

One of the central themes of this paper is the different status enjoyed by $k$ dependent and $k$-independent diffeomorphisms. We saw that the group of gauge transformations consists of the $k$-independent ones only and that this is one of the reasons why the effective field equations cannot completely determine the gauge invariant, i.e. "physical" contents of the family $\left\{\left\langle g_{\mu \nu}\right\rangle_{k}\right\}$. Depending on whether Killing vectors are $k$-independent or not they either implement a symmetry on the manifold of physical events or they are "anomalous".

We also discussed the possibility of assigning an intrinsic length to objects living in a QEG spacetime, defined as the proper length of an object when observed by a 
"microscope" which can just resolve it, and we investigated under which conditions this can be a meaningful notion.

\section{Acknowledgements}

M. R. would like to thank M. Niedermaier and R. Percacci for helpful discussions. He is also grateful to the Albert Einstein Institute for the hospitality extended to him while this work was in progress.

\section{References}

[1] M. Reuter, Phys. Rev. D 57 (1998) 971 and hep-th/9605030.

[2] D. Dou and R. Percacci, Class. Quant. Grav. 15 (1998) 3449.

[3] O. Lauscher and M. Reuter, Phys. Rev. D 65 (2002) 025013 and hep-th/0108040.

[4] M. Reuter and F. Saueressig, Phys. Rev. D 65 (2002) 065016 and hep-th/0110054.

[5] O. Lauscher and M. Reuter, Phys. Rev. D 66 (2002) 025026 and hep-th/0205062.

[6] O. Lauscher and M. Reuter, Class. Quant. Grav. 19 (2002) 483 and hep-th/0110021.

[7] O. Lauscher and M. Reuter, Int. J. Mod. Phys. A 17 (2002) 993 and hep-th/0112089.

[8] W. Souma, Prog. Theor. Phys. 102 (1999) 181.

[9] R. Percacci and D. Perini, Phys. Rev. D 67 (2003) 081503; Phys. Rev. D 68 (2003) 044018; D. Perini, Nucl. Phys. Proc. Suppl. 127 C (2004) 185. 
[10] A. Codello and R. Percacci, hep-th/0607128.

[11] M. Reuter and F. Saueressig, Phys. Rev. D 66 (2002) 125001 and hep-th/0206145 Fortschr. Phys. 52 (2004) 650 and hep-th/0311056.

[12] D. Litim, Phys. Rev. Lett. 92 (2004) 201301; hep-th/0606044; P. Fischer and D. Litim, hep-th/0602203; hep-th/0606135.

[13] A. Bonanno, M. Reuter, JHEP 02 (2005) 035 and hep-th/0410191.

[14] For a review see: O. Lauscher and M. Reuter, hep-th/0511260.

[15] R. Percacci and D. Perini, Class. Quant. Grav. 21 (2004) 5035 and hep-th/0401071.

[16] R. Percacci, hep-th/0409199.

[17] S. Weinberg in General Relativity, an Einstein Centenary Survey, S.W. Hawking and W. Israel (Eds.), Cambridge University Press (1979);

S. Weinberg, hep-th/9702027.

[18] For a review see: M. Niedermaier and M. Reuter, Living Reviews in Relativity, to appear.

[19] P. Forgács and M. Niedermaier, hep-th/0207028;

M. Niedermaier, JHEP 12 (2002) 066; Nucl. Phys. B 673 (2003) 131; preprint gr-qc/0610018.

[20] C. Wetterich, Phys. Lett. B 301 (1993) 90.

[21] M. Reuter and C. Wetterich, Nucl. Phys. B 417 (1994) 181, Nucl. Phys. B 427 (1994) 291, Nucl. Phys. B 391 (1993) 147, Nucl. Phys. B 408 (1993) 91; M. Reuter, Phys. Rev. D 53 (1996) 4430, Mod. Phys. Lett. A 12 (1997) 2777. 
[22] For a review see: J. Berges, N. Tetradis and C. Wetterich, Phys. Rep. 363 (2002) 223; C. Wetterich, Int. J. Mod. Phys. A 16 (2001) 1951.

[23] For a review see: C. Bagnuls and C. Bervillier, Phys. Rep. 348 (2001) 91; T.R. Morris, Prog. Theor. Phys. Suppl. 131 (1998) 395; J. Polonyi, Central Eur. J. Phys. 1 (2004) 1.

[24] J. Ambjørn, J. Jurkiewicz and R. Loll, Phys. Rev. Lett. 93 (2004) 131301.

[25] O. Lauscher and M. Reuter, JHEP 10 (2005) 050 and hep-th/0508202.

[26] A. Connes, hep-th/0608226; A.H. Chamseddine, A. Connes and M. Marcolli, hep-th/0610241.

[27] J. Ambjørn, J. Jurkiewicz and R. Loll, Phys. Lett. B 607 (2005) 205.

[28] J. Ambjørn, J. Jurkiewicz and R. Loll, Phys. Rev. Lett. 95 (2005) 171301; Phys. Rev. D 72 (2005) 064014; Contemp. Phys. 47 (2006) 103.

[29] A. Bonanno and M. Reuter, Phys. Rev. D 62 (2000) 043008 and hep-th/0002196; Phys. Rev. D 73 (2006) 083005 and hep-th/0602159; Phys. Rev. D 60 (1999) 084011 and gr-qc/9811026.

[30] A. Bonanno and M. Reuter, Phys. Rev. D 65 (2002) 043508 and hep-th/0106133;

M. Reuter and F. Saueressig, JCAP 09 (2005) 012 and hep-th/0507167.

[31] A. Bonanno and M. Reuter, Phys. Lett. B 527 (2002) 9 and astro-ph/0106468; Int. J. Mod. Phys. D 13 (2004) 107 and astro-ph/0210472.

[32] E. Bentivegna, A. Bonanno and M. Reuter, JCAP 01 (2004) 001 and astro-ph/0303150. 
[33] A. Bonanno, G. Esposito and C. Rubano, Gen. Rel. Grav. 35 (2003) 1899; Class. Quant. Grav. 21 (2004) 5005; A. Bonanno, G. Esposito, C. Rubano and P. Scudellaro, Class. Quant. Grav. 23 (2006) 3103; preprint gr-qc/0610012.

[34] M. Reuter and H. Weyer, Phys. Rev. D 69 (2004) 104022 and hep-th/0311196.

[35] M. Reuter and H. Weyer, Phys. Rev. D 70 (2004) 124028 and hep-th/0410117.

[36] M. Reuter and H. Weyer, JCAP 12 (2004) 001 and hep-th/0410119.

[37] F. Girelli, S. Liberati, R. Percacci, C. Rahmede, gr-qc/0607030.

[38] J. Moffat, JCAP 05 (2005) 003 and astro-ph/0412195; J.R. Brownstein and J. Moffat, Astrophys. J. 636 (2006) 721; Mon. Not. Roy. Astron. Soc. 367 (2006) 527.

[39] L.F. Abbott, Nucl. Phys. B 185 (1981) 189; B.S. DeWitt, Phys. Rev. 162 (1967) 1195; M.T. Grisaru, P. van Nieuwenhuizen and C.C. Wu, Phys. Rev. D 12 (1975) 3203; D.M. Capper, J.J. Dulwich and M. Ramon Medrano, Nucl. Phys. B 254 (1985) 737; S.L. Adler, Rev. Mod. Phys. 54 (1982) 729.

[40] B. Mandelbrot, The Fractal Geometry of Nature, Freeman, New York (1983).

[41] D. ben-Avraham and S. Havlin, Diffusion and reactions in fractals and disordered systems, Cambridge University Press, Cambridge (2004).

[42] H. Kawai, M. Ninomiya, Nucl. Phys. B 336 (1990) 115;

R. Floreanini and R. Percacci, Nucl. Phys. B 436 (1995) 141;

I. Antoniadis, P.O. Mazur and E. Mottola, Phys. Lett. B 444 (1998) 284. 
[43] G.M. Shore, Nucl. Phys. B460 (1996) 379.

[44] M. Reuter and J. Schwindt, JHEP 0601 (2006) 070 and hep-th/0511021; preprint hep-th/0610064.

[45] S. Weinberg, Gravitation and Cosmology, J.Wiley, New York, 1972.

[46] J.F. Donoghue, Phys. Rev. Lett. 72 (1994) 2996; Phys. Rev. D50 (1994) 3874

[47] N.E.J. Bjerrum-Bohr, Phys. Rev. D66 (2002) 084023; N.E.J. BjerrumBohr, J.F. Donoghue and B.R. Holstein, Phys. Rev. D67 (2003) 084033; Phys. Rev. D68 (2003) 084005.

[48] R.M. Corless et al., Advances in Computational Mathematics 5 (1996) 329. 